\title{
Generalized 't Hooft anomalies on non-spin manifolds
}

\section{Mohamed M. Anber ${ }^{a}$ and Erich Poppitz ${ }^{b}$}

\author{
${ }^{a}$ Department of Physics, Lewis and Clark College, \\ Portland, OR 97219, U.S.A. \\ ${ }^{b}$ Department of Physics, University of Toronto, \\ Toronto, ON M5S 1A\%, Canada \\ E-mail: manber@lclark.edu, poppitz@physics.utoronto.ca
}

\begin{abstract}
We study the mixed anomaly between the discrete chiral symmetry and general baryon-color-flavor (BCF) backgrounds in $\mathrm{SU}\left(N_{c}\right)$ gauge theories with $N_{f}$ flavors of Dirac fermions in representations $\mathcal{R}_{c}$ of $N$-ality $n_{c}$, formulated on non-spin manifolds. We show how to study these theories on $\mathbb{C P}^{2}$ by turning on general BCF fluxes consistent with the fermion transition functions. We consider several examples in detail and argue that matching the anomaly on non-spin manifolds places stronger constraints on the infrared physics, compared to the ones on spin manifolds (e.g. $\mathbb{T}^{4}$ ). We also show how to consistently formulate various chiral gauge theories on non-spin manifolds.
\end{abstract}

Keywords: Anomalies in Field and String Theories, Discrete Symmetries, Global Symmetries

ARXIV EPRINT: 2002.02037 


\section{Contents}

1 Introduction

2 Baryon-Color-Flavor (BCF) 't Hooft fluxes on $\mathbb{C P}^{2}$ for vector-like theories

2.1 Vector-like theories 3

2.2 Generalized 't Hooft fluxes on $\mathbb{C P}^{2}$

2.3 Comment on chiral theories and the Standard Model with $\nu_{R} \quad 6$

3 Anomalies in the background of BCF fluxes on $\mathbb{C P}^{2} \quad 7$

3.1 Examples 10

$\begin{array}{lll}3.1 .1 & \text { QCD }(\text { adj }) & 10\end{array}$

3.1.2 $\mathrm{SU}(6)$ with a Dirac fermion in the two-index anti-symmetric representation

3.1.3 $\mathrm{SU}(4 k+2)$ with fermions in the two-index (anti)-symmetric representation

3.2 Comments on future studies

A Some useful formulae for $\mathbb{C P}^{2}$

B Gauge fields and fermions on $\mathbb{C P}^{2}$

\section{Introduction}

Anomaly matching conditions provide a rare exact constraint on the infrared (IR) behavior of strongly coupled gauge theories [1]. To study the matching of anomalies, one probes the theory with nondynamical (background) gauge fields for its anomaly-free global symmetries. Any violation of the background gauge invariance due to the resulting 't Hooft anomalies should exactly match between the ultraviolet (UV), usually free, and IR descriptions of the theory. In the past, these consistency conditions have been applied to " 0 -form" symmetries, acting on local fields. For example, anomaly matching was instrumental in the study of models of quark and lepton compositeness in the 1980s (see the review [2]) or of Seiberg duality in the 1990s [3].

Recently, it was realized that the scope of anomaly matching is significantly wider than originally thought [4-6]. Turning on general background fields - corresponding to global, spacetime, continuous, discrete, 0-form, or higher-form symmetries, consistent with their faithful action - was argued to lead to new UV-IR anomaly matching conditions. We refer to them as "generalized 't Hooft anomalies." The study of these generalized 
anomalies is a currently active area of research with contributions coming from the highenergy, condensed matter, and mathematical communities. We do not claim to be in command of all points of view and only give a list of references written from a (largely) high-energy physics perspective and pertaining to theories somewhat similar to the ones discussed in this paper [7-19].

Summary: We continue our study [20] of the generalized 't Hooft anomalies in $\mathrm{SU}\left(N_{c}\right)$ gauge theories with $N_{f}$ flavors of Dirac fermions in representations $\mathcal{R}_{c}$ of $N_{c}$-ality $n_{c}$. These theories have exact global discrete chiral symmetries. Considering these theories on $\mathbb{T}^{4}$ and turning on the most general 't Hooft flux [21] backgrounds for the global symmetries, consistent with their faithful action, we found a mixed anomaly between the discrete chiral symmetry and the $\mathrm{U}\left(N_{f}\right) / \mathbb{Z}_{N_{c}}$ baryon-color-flavor, or "BCF", background. We showed that matching this $\mathrm{BCF}$ anomaly imposes new constraints on possible scenarios for IR physics, in addition to those imposed by the "traditional" 0-form 't Hooft anomalies. When these theories are coupled to axions, the axion theory is also constrained by anomalies [22].

In this paper, we consider the fate of the BCF anomalies in the same class of theories, but now formulated on non-spin manifolds. We are motivated by the study of QCD(adj) [23], which showed that 't Hooft anomalies in theories with fermions on non-spin backgrounds impose additional constraints. It is known that manifolds that do not permit a spin structure $[24,25]$ can accommodate theories with fermions, but only if appropriate gauge fluxes are turned on [26]. These fluxes can correspond to dynamical or background fields, as in the recent studies [23, 27-29]. We focus on the canonical example of non-spin manifold, $\mathbb{C P}^{2}$. It has the advantage of allowing for an explicit (and pedestrian ${ }^{1}$ ) discussion of the salient points. We describe in detail how to turn on background $\mathrm{U}\left(N_{f}\right) / \mathbb{Z}_{N_{c}}$ fluxes on $\mathbb{C P}^{2}$ and derive the resulting BCF anomaly on non-spin backgrounds. The final result of our analysis is that the BCF anomaly matching conditions on $\mathbb{C P}^{2}$ are equal or stronger than those obtained on $\mathbb{T}^{4}$. We use several examples to show that the BCF anomaly on $\mathbb{C P}^{2}$ further constrains various scenarios for the IR dynamics.

Organization of this paper: in section 2.1, we define the class of theories we study. In section 2.2, inviting the reader to also consult appendices A and B, we explain how to turn on 't Hooft fluxes on $\mathbb{C P}^{2}$ for the baryon, color, and flavor gauge fields, consistent with the faithful action of the global symmetries in the representation $\mathcal{R}_{c}$.

In section 2.3 , we temporarily divert to show how to put chiral gauge theories in non-spin backgrounds; however, we leave their study for the future.

In section 3, we study the mixed 't Hooft anomalies of the discrete chiral symmetry with the BCF fluxes on $\mathbb{C P}^{2}$, discuss the conditions imposed on the IR spectrum, and compare with the case of $\mathbb{T}^{4}$ studied previously.

\footnotetext{
${ }^{1}$ See appendix A for details of $\mathbb{C P}^{2}$ and appendix B for an explicit description of how to consistently turn on 't Hooft fluxes on $\mathbb{C P}^{2}$ in theories with fermions in general representations. This discussion complements the more abstract mathematical descriptions existing in the literature. At the end, the anomaly depends only on topological information. However, considering explicit gauge and gravity backgrounds ('t Hooft fluxes in a $\mathbb{C P}^{2}$ background) provides a more "pedestrian" route to see the anomaly, which might be more familiar for many physicists.
} 
In section 3.1, we present several examples. In section 3.1.1 we discuss QCD(adj). Our intention is to use the present study to investigate the various scenarios for IR behavior, whose consistency has been recently elaborated upon in [16, 19, 23, 28, 30-32]. In section 3.1.2, we study an SU(6) gauge theory with a single Dirac flavor in the two-index antisymmetric representation and, in section 3.1.3, its generalization to $\mathrm{SU}(4 k+2)$ with a single flavor of two-index symmetric or antisymmetric representations. In both cases, we argue that scenarios for IR physics consistent with the 0-form 't Hooft anomalies are further constrained by studying them on $\mathbb{C P}^{2}$. In particular, we focus on exotic phases ${ }^{2}$ with massless composite fermions, and argue that the TQFT which must accompany the massless composites has to reproduce a more restrictive anomaly on $\mathbb{C P}^{2}$.

Appendices $\mathrm{A}$ and $\mathrm{B}$ contain many relevant formulae regarding $\mathbb{C P}^{2}$ and fermions. At the end of appendix B, we find several classes of theories which can be formulated on $\mathbb{C P}^{2}$ by turning on of only dynamical gauge backgrounds, i.e. by only modifying the gauge bundles summed over. These gauge theories share a feature common with examples discussed in $[27,28]$ : they have only bosonic gauge invariant operators and can be taught as emergent descriptions near quantum critical points of purely bosonic systems.

\section{Baryon-Color-Flavor (BCF) 't Hooft fluxes on $\mathbb{C P}^{2}$ for vector-like the- ories}

In this section, we describe in great detail (in conjunction with appendices A and B) how to introduce background fluxes in the baryon-number, color, and flavor directions on $\mathbb{C P}^{2}$. We carry out our construction for vector-like theories. However, this setup can be easily adapted for chiral theories (such as the Standard Model), as we show at the end of this section.

\section{$2.1 \quad$ Vector-like theories}

We consider $\mathrm{SU}\left(N_{c}\right)$ gauge theories with $N_{f}$ flavors of Dirac fermions transforming in a representation $\mathcal{R}^{c}$ of $\mathrm{N}$-ality $n_{c}{ }^{3}$ The gauge group that faithfully acts on the fermions is $\frac{\mathrm{SU}\left(N_{c}\right)}{\mathbb{Z}_{p}}$, where $p=\operatorname{gcd}\left(N_{c}, n_{c}\right)$; thus, the fermions are charged under a $\mathbb{Z}_{\frac{N_{c}}{p}}$ subgroup of the center of $\mathrm{SU}\left(N_{c}\right)$. After modding out the redundant symmetries, we find that the 0 -form global symmetry of the theory is

$$
G^{\text {global }}=\frac{\mathrm{SU}\left(N_{f}\right)_{L} \times \mathrm{SU}\left(N_{f}\right)_{R} \times \mathrm{U}(1)_{B} \times \mathbb{Z}_{2 \operatorname{dim}\left(\mathcal{R}^{f}\right) T_{\mathcal{R}^{c}}},}{\mathbb{Z}_{\frac{N_{c}}{p}} \times \mathbb{Z}_{N_{f}} \times \mathbb{Z}_{2}},
$$

where $T_{\mathcal{R}}$ is the Dynkin index of the representation, $\mathcal{R}$ and $\operatorname{dim}(\mathcal{R})$ is its dimension. Here, we assume that $\mathbb{Z}_{2 \operatorname{dim}\left(\mathcal{R}^{f}\right) T_{\mathcal{R}^{c}}}$ is a genuine symmetry of the theory; thus, it cannot be absorbed in the continuous part of $G^{\text {global }}$ (this can be checked on a case by case basis). $\mathbb{Z}_{2}$ above denotes fermion number and $\mathbb{Z}_{\frac{N_{c}}{p}}$ is in the center of $\mathrm{SU}\left(N_{c}\right)$.

\footnotetext{
${ }^{2}$ The examples of sections 3.1.2 and 3.1.3 were also studied in refs. [16, 19], which argued that an IR gapped phase with unbroken global symmetries cannot occur.

${ }^{3}$ The $N$-ality of a representation $\mathcal{R}$ of $\mathrm{SU}(N)$ is the number of boxes of the Young tableau of $\mathcal{R}$ modulo $N$.
} 
In addition, the theory has a 1 -form center symmetry $\mathbb{Z}_{p}^{(1)}$ that acts on non-contractible Wilson loops, provided that $\operatorname{gcd}\left(N_{c}, n_{c}\right)=p>1$. Notice that the ultraviolet fermions are taken to transform in the defining representation of the flavor group $\mathrm{SU}\left(N_{f}\right)$, and hence, we should use $n_{f}=1$. Nevertheless, we keep the $N$-ality of the fermions under $\mathrm{SU}\left(N_{f}\right)$ an arbitrary integer for the sake of generality.

\subsection{Generalized 't Hooft fluxes on $\mathbb{C P}^{2}$}

Next, we turn on 't Hooft fluxes (twists) in the baryon-number, color, and flavor directions, which are compatible with $\mathbb{C P}^{2}$ and at the same time lead to consistent transition functions. See appendix A for a collection of relevant formulae for $\mathbb{C P}^{2}$.

We first address the compatibility condition. As we point out in appendix B, background gauge fields (both abelian and nonabelian) on $\mathbb{C P}^{2}$ need to be (anti)self-dual, otherwise they will have a nonvanishing energy-momentum, and hence, backreact on the manifold. In order to achieve the (anti)self-duality, we take the gauge fields to be proportional to the Kähler 2-form $K$ of $\mathbb{C P}^{2}$, eqs. (A.2), (A.9):

$$
T^{a} F^{a} \sim T^{a} C^{a} K
$$

where $T^{a}$ stands for the color, flavor, or baryon-number generators, and $C^{a}$ are constants that will be determined momentarily.

Second, we come to the problem of defining a consistent gauge theory with matter fields on a manifold $\mathcal{M}$. Let $G$ be a direct product of semi-simple Lie groups and $\Psi$ a fermionic matter field transforming under specific representations of $G$. A quantum field theory of $\Psi$ is described in terms of a collection of covers $\left\{U_{i}\right\}$ of $\mathcal{M}$ (in $\left\{U_{i}\right\}, \Psi$ is denoted $\Psi_{i}$ ), along with transition functions $g_{i j} \in G$, defined on the overlap $U_{i} \cap U_{j}$ and relating $\Psi_{i}$ to $\Psi_{j}$

$$
\Psi_{i}=\mathcal{G}_{i j} \Psi_{j}
$$

where

$$
\mathcal{G}_{i j}=g_{i j}^{B} g_{i j}^{\mathcal{R}^{c}} g_{i j}^{\mathcal{R}^{f}} g_{i j}^{L},
$$

such that $g_{i j}^{B, \mathcal{R}^{c}, \mathcal{R}^{f}}$ are the transition functions of the baryon, color, and flavor groups, while $g_{i j}^{L}$ is the transition function associated to the spacetime Lorentz group. The matter field in general will transform under representation $\mathcal{R}^{c}$ of the color group and representation $\mathcal{R}^{f}$ of the flavor group. However, only the $\mathrm{N}$-ality of the representations will matter in what follows. Consistency requires that the transition functions satisfy the cocycle conditions on the triple overlap $U_{i} \cap U_{j} \cap U_{k}$ :

$$
\mathcal{G}_{i j} \mathcal{G}_{j k} \mathcal{G}_{k i}=1
$$

The above cocycle condition does not necessary imply that the strong conditions $g_{i j}^{a} g_{j k}^{a} g_{k i}^{a}=$ 1 should be met for each of the transition functions in (2.4), where $a$ refers to the baryonnumber, flavor, color, or Lorentz groups. 
Let $g_{i j}^{c}$ and $g_{i j}^{f}$ be the transition functions in the defining representations of the color and flavor groups, respectively. One, then, may relax the condition (2.5) to the following set of conditions

$$
\begin{aligned}
& g_{i j}^{c} g_{j k}^{c} g_{k i}^{c}=e^{i \frac{2 \pi}{N_{c}} n_{i j k}^{(c)}}, \quad g_{i j}^{f} g_{j k}^{f} g_{k i}^{f}=e^{i \frac{2 \pi}{N_{f}} n_{i j k}^{(f)}}, \\
& g_{i j}^{B} g_{j k}^{B} g_{k i}^{B}=e^{-i \pi-i n_{c} \frac{2 \pi}{N_{c}} n_{i j k}^{(c)}-i n_{f} \frac{2 \pi}{N_{f}} n_{i j k}^{(f)}}
\end{aligned}
$$

on the triple overlap. In this expression $n_{c}\left(n_{f}\right)$ is the color (flavor) N-ality, $n_{i j k}^{(c)}\left(n_{i j k}^{(f)}\right)$ are integers modulo $N_{c}\left(N_{f}\right)$, while the factor $e^{-i \pi}$ that appears in the last cocycle condition cancels the minus sign arising from parallel transporting the spinor fields around appropriate closed paths in $\mathbb{C P}^{2}$, see appendix B and [24-26].

Thus, the $\mathrm{U}(1)_{B}$ bundle provides the flux that is necessary to render the fermions well-defined on the non-spin manifold. As a side remark, we note that this is by no means is the unique choice to put spinors on $\mathbb{C P}^{2}$ : one could also use the fluxes in the color (or flavor) directions to perform the same job. Examples of using only gauge backgrounds (i.e. modifying only the gauge bundles being summed over in the path integral) are known in the literature [27-29] and we give a few more at the end of appendix B; a common feature of gauge theories where this can be done is their possible interpretation as emergent descriptions near quantum critical points in theories of only bosons [28].

The consistency conditions (2.5) or (2.6) guarantee that the Dirac index will always be an integer. Since the Dirac index counts the number of the fermion zero modes in a given gauge/gravity background, the integrality of the index is a necessary condition for the consistency of a given theory in the background of baryon-color-flavor 't Hooft fluxes in $\mathbb{C P}^{2}$. The integrality of the index will be manifest in all the examples we discuss in this paper.

Having all the ingredients necessary to turn on compatible fluxes on non-spin manifolds, we now choose the color and flavor fluxes in the Cartan directions of the respective groups. Using (2.2) we write:

$$
\begin{aligned}
T^{a(c)} F^{a(c)} & =\boldsymbol{H}^{c} \cdot \boldsymbol{\nu}^{c} m^{c} K, \\
T^{a(f)} F^{a(f)} & =\boldsymbol{H}^{f} \cdot \boldsymbol{\nu}^{f} m^{f} K, \\
F^{B} & =\left(\frac{1}{2}+\frac{n^{c}}{N_{c}} m^{c}+\frac{n^{f}}{N_{f}} m^{f}\right) K .
\end{aligned}
$$

Here $\boldsymbol{H}^{c / f}$ are the fundamental representation Cartan generators of $\mathrm{SU}\left(N_{c / f}\right)$, obeying $\operatorname{tr}\left[H^{a} H^{b}\right]=\delta^{a b}$, and $\boldsymbol{\nu}$ are the weights of the corresponding defining representation, $\boldsymbol{\nu}^{a} \cdot \boldsymbol{\nu}^{b}=\delta^{a b}-\frac{1}{N}$ (where $N$ stands for $N_{c}$ or $N_{f}$ ). The fluxes (2.7), with integer $m^{c}$ and $m^{f}$, are compatible with the cocycle conditions (2.6), see (B.7), and the Dirac index is integer in their background. The topological charges are given by

$$
Q=\frac{1}{8 \pi^{2}} \int \operatorname{tr}[F \wedge F]
$$


Then, substituting (2.7) into (2.8) and using $\int_{\mathbb{C P}^{2}} \frac{K \wedge K}{8 \pi^{2}}=\frac{1}{2}$, we find:

$$
\begin{aligned}
Q^{c} & =\frac{\left(m^{c}\right)^{2}}{2}\left(1-\frac{1}{N_{c}}\right), \quad Q^{f}=\frac{\left(m^{f}\right)^{2}}{2}\left(1-\frac{1}{N_{f}}\right), \\
Q^{B} & =\frac{1}{2}\left(\frac{1}{2}+\frac{n^{c}}{N_{c}} m^{c}+\frac{n^{f}}{N_{f}} m^{f}\right)^{2} .
\end{aligned}
$$

Adding to this list the gravitational topological charge of $\mathbb{C P}^{2}$

$$
Q^{G}=\frac{1}{192 \pi^{2}} \int \operatorname{tr}[R \wedge R]=-\frac{1}{8}
$$

we finally obtain the Dirac index:

$$
\mathcal{J}_{D}=T_{\mathcal{R}^{c}} \operatorname{dim}_{\mathcal{R}^{f}} Q^{c}+T_{\mathcal{R}^{f}} \operatorname{dim}_{\mathcal{R}^{c}} Q^{f}+\operatorname{dim}_{\mathcal{R}^{f}} \operatorname{dim}_{\mathcal{R}^{c}}\left(Q^{B}+Q^{G}\right),
$$

which is an integer for all the examples we consider below.

Before moving to examples, it is instructive to compare and contrast the above results with the BCF fluxes on the four-torus $\mathbb{T}^{4}$ that we considered before [20]. $\mathbb{C P}^{2}$ has one two-cycle $\mathbb{C P}^{1}$, and hence, we were able to turn on fluxes along this single cycle (the color and flavor fluxes are labeled by $m^{c, f}$ in (2.9)). In contrast, $\mathbb{T}^{4}$ has six two-cycles (it suffices to turn on fluxes in the 1-2 or 3-4 planes, respectively, hence we have two integers $m_{12}$ and $m_{34}$ that label the fluxes). ${ }^{4}$ Since there are more ways to turn on fluxes on $\mathbb{T}^{4}$ compared to $\mathbb{C P}^{2}$, this may imply that putting the theory on $\mathbb{T}^{4}$ can give us more constraining conditions on the IR spectrum. We will see in the next section that this is not true: although $\mathbb{C P}^{2}$ has only one cycle, it always imposes conditions that are either stronger or at least as strong as the conditions we obtain by putting the theory on $\mathbb{T}^{4}$.

\subsection{Comment on chiral theories and the Standard Model with $\nu_{R}$}

Here, we slightly divert from our main presentation to note, for the sake of completeness, that by turning on global anomaly-free U(1) fluxes, chiral gauge theories can also be formulated on non-spin manifolds.

As an example, consider an SU(5) gauge theory with $\mathbf{5}^{*}$ and $\mathbf{1 0}$ left-handed Weyl fermions: $:^{5} \lambda$ in the anti-fundamental and $\psi$ in the two-index anti-symmetric representations. This theory has an anomaly-free global U(1) that acts on the fermions as $\psi \rightarrow e^{i 2 \pi \alpha} \psi$ and $\lambda \rightarrow e^{-i 2 \pi(3 \alpha)} \lambda$. Then, one can easily check that the flux

$$
\begin{aligned}
T^{a(c)} F^{a(c)} & =\boldsymbol{H}^{c} \cdot \boldsymbol{\nu}^{c} m^{c} K, \\
F^{\mathrm{U}(1)} & =-\left(\frac{1}{2}+\frac{2}{5} m^{c}\right) K,
\end{aligned}
$$

\footnotetext{
${ }^{4}$ For the sake of completeness, we give $Q^{c, f, B}$ on $\mathbb{T}^{4}[20]$ :

$$
\begin{aligned}
Q^{c} & =m_{12}^{c} m_{34}^{c}\left(1-\frac{1}{N_{c}}\right), \quad Q^{f}=m_{12}^{f} m_{34}^{f}\left(1-\frac{1}{N_{f}}\right), \\
Q^{B} & =\left(n_{c} \frac{m_{12}^{c}}{N_{c}}+n_{f} \frac{m_{12}^{f}}{N_{f}}\right)\left(n_{c} \frac{m_{34}^{c}}{N_{c}}+n_{f} \frac{m_{34}^{f}}{N_{f}}\right) .
\end{aligned}
$$
}

${ }^{5}$ For a discussion of its conjectured IR dynamics, see [33]. 
is consistent with the cocycle condition (2.5) for both $\psi$ and $\lambda$. This can be seen by considering the consistency condition (B.7) on $\mathbb{C P}^{2}$ for fermions in these two representations, taking into account their different $\mathrm{U}(1)$ charges and $\mathrm{SU}(5)$ representations. One can also check the consistency by calculating the Dirac indices for both $\psi$ and $\lambda$ : using $Q^{c}=\frac{1}{2}\left(m^{c}\right)^{2}\left(1-\frac{1}{5}\right)$ and $Q^{\mathrm{U}(1)}=\frac{1}{2}\left(\frac{1}{2}+\frac{2}{5} m^{c}\right)^{2}$, we obtain

$$
\begin{aligned}
& \mathcal{J}_{\psi}=T_{\psi} Q^{c}+\operatorname{dim}_{\psi}\left(Q^{\mathrm{U}(1)}-\frac{1}{8}\right)=2 m^{c}\left(1+m^{c}\right), \\
& \mathcal{J}_{\lambda}=T_{\lambda} Q^{c}+\operatorname{dim}_{\lambda}\left((3)^{2} Q^{\mathrm{U}(1)}-\frac{1}{8}\right)=5+9 m^{c}+4\left(m^{c}\right)^{2},
\end{aligned}
$$

which are integers. Notice that the total number of upper minus lower SU(5) indices of the zero modes is a multiple of 5 (and the total number of zero modes is even for odd $m_{c}$ ), so that a gauge invariant "t Hooft vertex" using the zero modes can be written.

Let us also mention that the Standard Model can be formulated on a non-spin manifold, provided that right-handed neutrinos are added. ${ }^{6}$ In this case one can turn on a fractional flux in the global $\mathrm{U}(1)_{B-L}$ in order to cancel the $e^{i \pi}$ ambiguity that results from putting the quarks and leptons on $\mathbb{C P}^{2}$. By computing the indices, as above, it is easy to see that gauge and Lorentz invariant terms can be constructed out of the zero modes. The $\mathrm{U}(1)_{B-L}$ can further be promoted to a gauge symmetry, broken by a charge- 2 Higgs. For related discussions see [34, 35] as well as the remarks on the Spin(10) grand unified theory in [27].

In the two examples mentioned in this section, formulating the theory on $\mathbb{C P}^{2}$ does not lead to new 't Hooft anomalies of the type discussed here, as these theories only have continuous chiral symmetries whose anomalies are matched irrespective of the integrality of the topological charges. ${ }^{7}$ Further study of chiral theories is left for the future.

\section{Anomalies in the background of BCF fluxes on $\mathbb{C P}^{2}$}

We now return back to our main theme and examine the fate of the axial symmetries of vector-like theories as we put them in the background of BCF fluxes. In order to reduce notational clutter, we assume that the theory enjoys a genuine discrete $\mathbb{Z}_{q_{g}}$ axial global symmetry, which becomes anomalous in the background of BCF fluxes. We denote by $D^{c, f, B, G}$ the anomaly coefficients that accompany the color, flavor, baryon-number, and gravitational topological charges. The UV values of these coefficients, $D_{\mathrm{UV}}^{c}, D_{\mathrm{UV}}^{f}, D_{\mathrm{UV}}^{B}, D_{\mathrm{UV}}^{G}$, are equal to twice the pre-factors that multiply $Q^{c, f, B, G}$, respectively, in the Dirac index (2.11): these are group-theoretical values and they do not depend on whether we turn on integer or fractional fluxes or whether we put the theory on spin or non-spin manifolds. To summarize, upon performing a global $\mathbb{Z}_{q_{g}}$ axial transformation on the fermions, the UV partition function acquires the phase

$$
\left.\mathcal{Z}_{\mathrm{UV}}\right|_{\mathbb{Z}_{q_{g}}} \rightarrow \mathcal{Z} e^{i \frac{2 \pi}{q_{g}}\left(D_{\mathrm{UV}}^{c} Q^{c}+D_{\mathrm{UV}}^{f} Q^{f}+D_{\mathrm{UV}}^{B} Q^{B}+D_{\mathrm{UV}}^{G} Q^{G}\right)}=\mathcal{Z} e^{i \frac{2 \pi}{q_{g}} 2 \mathcal{J}_{D}}
$$

\footnotetext{
${ }^{6}$ In the absence of right-handed neutrinos one finds that $\mathrm{U}(1)_{B-L}$ is broken by gravitational instantons.

${ }^{7}$ See $[20]$ for a lucid explanation why continuous chiral symmetry transformations in BCF backgrounds do not impose further constraints.
} 
where $\mathcal{J}_{D}$ is the Dirac index (2.11). This phase is a manifestation of a 't Hooft anomaly between the 0 -form $\mathbb{Z}_{q_{g}}$ symmetry and a general BCF background.

Now, we assume that the 0-form ("traditional") 't Hooft anomalies, which correspond to integer values of $Q^{c, f, B, G}$, can be matched by a set of fermion composites deep in the IR on a spin manifold. Upon performing a $\mathbb{Z}_{q_{g}}$ transformation in the IR, the partition function transforms as

$$
\left.\mathcal{Z}_{\mathrm{IR}}\right|_{\mathbb{Z}_{q_{g}}} \rightarrow \mathcal{Z} e^{i \frac{2 \pi}{q_{g}}\left(D_{\mathrm{IR}}^{c} Q^{c}+D_{\mathrm{IR}}^{f} Q^{f}+D_{\mathrm{IR}}^{B} Q^{B}+D_{\mathrm{IR}}^{G} Q^{G}\right)}
$$

where $D_{\mathrm{IR}}^{c}, D_{\mathrm{IR}}^{f}, D_{\mathrm{IR}}^{B}, D_{\mathrm{IR}}^{G}$ are the anomaly coefficients computed using the IR spectrum of composites. Since we are matching a discrete anomaly, the coefficients $D^{c, f, B, G}$ need not be exactly matched between the UV and IR. Instead, $D^{c, f, B}$ are matched modulo $q_{g}$ :

$$
D_{\mathrm{UV}}^{c, f, B}-D_{\mathrm{IR}}^{c, f, B}=q_{g} \ell^{c, f, B},
$$

for integers $\ell^{c, f, B}$. The coefficients $D^{G}$ are matched only modulo $q_{g} / 2$ : there is an integer $\ell^{G}$ such that

$$
D_{\mathrm{UV}}^{G}-D_{\mathrm{IR}}^{G}=\frac{q_{g}}{2} \ell^{G} .
$$

This is true since the gravitational topological charge of a spin manifold is an even number. ${ }^{8}$

Now, we would like to check whether the same set of IR composite fermions can also match the BCF anomaly as we turn on fractional fluxes on a non-spin manifold. Before doing that, we first note that if a non-spin manifold admits an elementary spinor $\Psi$, then by virtue of (2.5) and (2.6) a composite of these spinors can always be defined. Also, one can easily see the spin-charge relation of the composites: a fermion (boson), made of an odd (even) number of $\Psi$, carries an odd (even) charge under $\mathrm{U}(1)_{B}$.

Thus, using (3.1), (3.2), (3.3), and (3.4), we obtain the matching condition:

$$
\frac{\left.\mathcal{Z}_{\mathrm{UV}}\right|_{\mathbb{Z}_{q_{g}}}}{\left.\mathcal{Z}_{\mathrm{IR}}\right|_{\mathbb{Z}_{q_{g}}}}=e^{i 2 \pi\left(\ell^{c} Q^{c}+\ell^{f} Q^{f}+\ell^{B} Q^{B}+\frac{\ell^{G}}{2} Q^{G}\right)}=1,
$$

or in other words

$$
\ell^{c} Q^{c}+\ell^{f} Q^{f}+\ell^{B} Q^{B}+\frac{\ell^{G}}{2} Q^{G} \in \mathbb{Z}
$$

for all fractional charges $Q^{c, f, B, G}$ given in (2.9) and (2.10). The condition (3.6) can be translated into the following set of conditions on $\ell^{c, f, B, G}$, which can be obtained by turning on and off the fluxes in the various directions:

$$
\begin{aligned}
& \quad \ell^{c} N_{c}\left(N_{c}-1\right)+\ell^{B} n_{c}\left(n_{c}+N_{c}\right) \in 2 N_{c}^{2} \mathbb{Z}, \\
& \ell^{f} N_{f}\left(N_{f}-1\right)+\ell^{B} n_{f}\left(n_{f}+N_{f}\right) \in 2 N_{f}^{2} \mathbb{Z}, \\
& \quad \ell^{c} N_{f}^{2} N_{c}\left(N_{c}-1\right)+\ell^{f} N_{c}^{2} N_{f}\left(N_{f}-1\right)+\ell^{B}\left(n_{c} N_{c} N_{f}^{2}+n_{f} N_{f} N_{c}^{2}\right) \\
& \quad+\ell^{B}\left(n_{c} N_{f}+n_{f} N_{c}\right)^{2} \in 2 N_{c}^{2} N_{f}^{2} \mathbb{Z}, \\
& 2 \ell^{B} \quad-\ell^{G} \in 16 \mathbb{Z} .
\end{aligned}
$$

\footnotetext{
${ }^{8}$ Notice that $q_{g}$ is an even number, since $\mathbb{Z}_{q_{g}}$ has to contain $\mathbb{Z}_{2}$ as a subgroup in any theory that preserves its Lorentz symmetry.
} 
The importance of the above conditions is as follows: if no integers $\ell^{c, f, B, G}$ that satisfy (3.7) can be found, then composite fermions cannot solely match the BCF anomaly. Thus, either the composites do not form in the IR, or they are accompanied by a partial breaking of $\mathbb{Z}_{q_{g}}$, due to some higher dimensional fermion condensate that leaves the continuous flavor symmetries intact, and/or an IR TQFT.

For example, setting ${ }^{9} \ell^{c}=1$, it is straightforward to check that no integers $\ell^{c, f, B, G}$ exist that satisfy (3.7) if $N_{f} \geq 2$ and one of the following two conditions are met:

$$
\begin{aligned}
& \operatorname{gcd}\left(N_{c}, N_{f}\right)>1, \\
& \operatorname{gcd}\left(N_{c}, n_{c}\right)>1 .
\end{aligned}
$$

We call the inequalities (3.8) the "no-go condition" on the composites (we stress that they apply provided that $N_{f} \geq 2$ and recall that $n_{f}=1$ ). In the special case $N_{f}=1$, one needs to replace (3.8) by other sets of conditions that we do not quote here; they can be checked on a case by case basis using the first and last conditions in (3.7).

Now a few comments are in order:

1. The first three conditions (3.7) are functions of $\ell^{c, f, B}$, while the fourth condition is a function of two variables only, $\ell^{G}$ and $\ell^{B}$. Therefore, if $\ell^{c, f, B}$ can be found to satisfy conditions $(i)$ to $(i i i)$, then it is always trivial to find $\ell^{G} \in \mathbb{Z}$ that satisfies condition $(i v)$.

2. Given 1 above, one expects that turning on gravitational background does not alter the conditions that are needed to find a set of composites in the IR matching all anomalies. At this point, it is instructive to compare the set of conditions ( $i$ ) to (iii) in (3.7) with those that result from turning on BCF fluxes on $\mathbb{T}^{4}$, as was considered before $^{10}[20]$. Although the two sets of conditions appear to be unrelated, they give the exact same no-go condition (3.8).

3. However, as we shall show in the examples in section 3.1, putting the theory on a nonspin manifold can give rise to a more restrictive phase in the partition function, and hence, imposes more constraints on the IR TQFT that accompanies the composites.

4. As in [13, 14], we can also turn on a $\mathrm{SU}\left(N_{f}\right)$ invariant mass term that breaks $\mathrm{SU}\left(N_{f}\right)_{L} \times \mathrm{SU}\left(N_{f}\right)_{R}$ down to the diagonal vector subgroup. We will take the mass to be smaller than the strong-coupling scale of the theory and also introduce a $\theta$ parameter. Now, we examine how the partition function transforms under a shift of $\theta$ by multiples of $2 \pi$, i.e., we ask whether the theory suffers a $\theta$ -

\footnotetext{
${ }^{9}$ Notice that gauge invariant composites have $\ell^{c}=1$ in the vectorlike theories we consider: using $D_{\mathrm{IR}}^{c}=0$, since the composites are color singlets, we have $\ell^{c}=\frac{D_{\mathrm{UV}}^{c}}{q_{g}}=\frac{T_{\mathcal{R}^{c}} \operatorname{dim}_{\mathcal{R}^{f}}}{T_{\mathcal{R}^{c}} \operatorname{dim}_{\mathcal{R}^{f}}}=1$.

${ }^{10}$ For the sake of completeness, we recall that the conditions $(3.7)$ are replaced on $\mathbb{T}^{4}$ by:
}

$$
N_{c} \ell^{c}-\ell^{B} n_{c}^{2} \in N_{c}^{2} \mathbb{Z}, \quad N_{f} \ell^{f}-\ell^{B} n_{f}^{2} \in N_{f}^{2} \mathbb{Z}, \quad \ell^{B} \in \mathcal{Q} \frac{N_{c} N_{f}}{n_{c} n_{f}} \mathbb{Z},
$$

where $\mathcal{Q}$ is the smallest integer that makes $\mathcal{Q} \frac{N_{c} N_{f}}{n_{c} n_{f}}$ an integer. 
periodicity anomaly. To this end, we introduce, in addition to the $\theta$ term, general background field dependent counter terms. The topological part of the Lagrangian becomes $\mathcal{L}_{\text {top. }}=\theta Q^{c}+\Theta_{f} Q^{f}+\Theta_{B} Q^{B}+\frac{\Theta_{G}}{2} Q^{G}$, where the coefficients of the counterterms, $\Theta_{f}, \Theta_{B}, \frac{\Theta_{G}}{2}$, are general real numbers. They can, however, depend on $\theta$ and we demand that they shift by $2 \pi \mathbb{Z}$ under $2 \pi r$ shifts of $\theta$, so that they do not destroy the $\theta$ periodicity in backgrounds with integer $Q^{c}, Q^{f}, Q^{B}$ and even $Q^{G}$. In other words, we have that under $\theta \rightarrow \theta+2 \pi r$ (where $r \in \mathbb{Z}$ ), $\Delta \mathcal{L}_{\text {top. }}=2 \pi r Q^{c}+2 \pi s Q^{f}+2 \pi t Q^{B}+\frac{2 \pi u}{2} Q^{G}$, where $s, t, u \in \mathbb{Z}$.

Finally, we ask whether the transformation of the counter terms can compensate for the phase of the partition function under shifts of $\theta$ in the BCF background fluxes on $\mathbb{C P}^{2}$, i.e., we demand that under $\theta \rightarrow \theta+2 \pi r, \mathcal{L}_{\text {top. }} \rightarrow \mathcal{L}_{\text {top. }}+\Delta \mathcal{L}_{\text {top. }}$, with $\Delta \mathcal{L}_{\text {top. }}=2 \pi \mathbb{Z}$. Carrying out this exercise, we find that the requirement $\Delta \mathcal{L}_{\text {top. }}=2 \pi \mathbb{Z}$ (the absence of a $\theta$-periodicity anomaly) is met for general BCF fluxes if and only if conditions (3.7) are satisfied after replacing $\ell^{c, f, B, G} \rightarrow r, s, t, u$. Therefore, the conditions that exclude massless composites are the exact same conditions that give rise to $\theta$-periodicity anomaly: they are given, for $N_{f} \geq 2$, by the same conditions (3.8) found earlier in [14]. The anomaly implies that as one varies $\theta$ between 0 and $2 \pi$, the IR theory should either have domain walls or an IR TQFT that saturates the anomaly.

\subsection{Examples}

In this section, we consider two examples of vector-like theories and check whether putting them on non-spin manifolds and turning on the most general background fluxes imposes further restrictions on various scenarios for their IR dynamics. Many aspects of what we find have been previously recognized in $[16,19,23,28,31]$, especially in the framework of QCD(adj), our first example below. Nonetheless, we include it in order to show how it fits in the present more general and explicit framework.

\subsubsection{QCD $(\mathrm{adj})$}

As a first example, we consider QCD(adj), an $\mathrm{SU}\left(N_{c}\right)$ Yang-Mills theory endowed with $N_{f}$ massless Dirac flavors in the adjoint representation. The Dirac fermion is equivalent to two undotted Weyl massless fermions $\psi, \tilde{\psi}$, both transforming in the adjoint representation. The global symmetry of this theory that we shall utilize is

$$
G^{\text {Global }} \supset \frac{\mathrm{SU}\left(N_{f}\right)_{L} \times \mathrm{SU}\left(N_{f}\right)_{R} \times \mathrm{U}(1)_{B} \times \mathbb{Z}_{4 N_{c} N_{f}}}{\mathbb{Z}_{N_{f}} \times \mathbb{Z}_{2}} \times \mathbb{Z}_{N_{c}}^{(1)},
$$

where we included the 1 -form $\mathbb{Z}_{N_{c}}^{(1)}$ center symmetry that acts on Polyakov loops. The massless Dirac theory above is equivalent to the theory of $2 N_{f}$ massless Weyl adjoints ${ }^{11} \lambda^{i}$, which has a larger global $\mathrm{SU}\left(2 N_{f}\right)$ chiral symmetry, containing the $\mathrm{SU}\left(N_{f}\right)_{L} \times \mathrm{SU}\left(N_{f}\right)_{R} \times$ $\mathrm{U}(1)_{B}$ shown above. While studying the BCF anomaly on non-spin manifolds, however, we shall make use of the backgrounds (2.7) for the symmetry (3.10).

\footnotetext{
${ }^{11}$ Here $i=1, \ldots, 2 N_{f}$ and all $\lambda^{i}$ are undotted $\operatorname{SL}(2, \mathbb{C})$ spinors.
} 
This class of theories has been extensively studied in the continuum [36-38] and on the lattice [39-47], for general theoretical interest, but also because it includes theories of interest for model building beyond the Standard Model. The usual lore is that these theories will either flow to an IR conformal field theory or break their global symmetries, including the discrete chiral symmetry $\mathbb{Z}_{4 N_{c} N_{f}}$. However, more exotic scenarios have recently been discussed in [23, 28, 30-32]. ${ }^{12}$

In [30], we conjectured that the theory with $N_{c}=2$ and a single $N_{f}=1$ Dirac fermion will form a massless composite, schematically given by $(\lambda)^{3}$, a doublet under the enhanced $\mathrm{SU}\left(2 N_{f}\right)=\mathrm{SU}(2)$ flavor symmetry, accompanied by the breaking $\mathbb{Z}_{8} \rightarrow \mathbb{Z}_{4}$, due to an $\mathrm{SU}(2)$ invariant four-fermion condensate. This IR scenario has to be supplemented by a TQFT that matches a mixed anomaly between the 0 -form discrete chiral and 1-form center symmetries on non-spin backgrounds [23], further studied in [16, 19, 31].

Another exotic scenario, applicable to all $N_{c}, N_{f}$, is the proposal of [32], where the IR phase of the theory contains $\left(N_{c}^{2}-1\right) \times 2 N_{f}$ massless fermions (essentially providing a gauge invariant copy of the UV fermion spectrum) which can be thought of as created by operators of the form:

$$
\mathcal{O}_{1}^{i}=\operatorname{tr}\left[F_{\mu \nu} \gamma^{\mu \nu} \lambda^{i}\right], \quad \ldots, \mathcal{O}_{N_{c}^{2}-1}^{i}=\operatorname{tr}[\underbrace{F_{\mu \alpha} \ldots F_{\rho \nu}}_{N_{c}^{2}-1} \gamma^{\mu \nu} \lambda^{i}] .
$$

This class of composites match all the 0-form anomalies. In addition, there is a TQFT that matches the discrete chiral-center anomaly. Clearly this is also required by the "no-go condition" (ii.) from (3.8) as $\operatorname{gcd}\left(N_{c}, n_{c}=N_{c}\right)=N_{c}>1$.

It will be instructive to check whether putting $\mathrm{QCD}(\mathrm{adj})$ on $\mathbb{C P}^{2}$ can impose further constraints on the above IR scenarios. To this end, we first examine the transformation of the partition function in the UV under the $\mathbb{Z}_{4 N_{c} N_{f}}$ discrete chiral symmetry. The index (2.11) is now given by

$$
\mathcal{J}_{D}=2 N_{c} N_{f} Q^{c}+\left(N_{c}^{2}-1\right) Q^{f}+N_{f}\left(N_{c}^{2}-1\right)\left(Q^{B}+Q^{G}\right),
$$

where $Q^{c, f, B}$ are given in (2.9) after setting $n_{c}=0$ and $n_{f}=1$. This index is always an integer for all $m^{c}$ and $m^{f}$, as can be easily checked. Then, under a $\mathbb{Z}_{4 N_{c} N_{f}}$ transformation the partition function acquires the phase ${ }^{13}$

$$
\left(\left.\mathcal{Z}_{\mathrm{UV}}\right|_{\mathbb{Z}_{4 N_{c} N_{f}}}\right)_{\mathbb{C P}^{2}} \rightarrow \mathcal{Z} e^{i \frac{2 \pi}{2 N_{c} N_{f}}\left[N_{f}\left(m^{c}\right)^{2}\left(N_{c}-1\right)+\left(N_{c}^{2}-1\right)\left(\frac{m^{f}\left(m^{f}+1\right)}{2}+m^{B} m^{f}+N_{f} \frac{m^{B}\left(m^{B}+1\right)}{2}\right)\right]}
$$

Thus, $\mathcal{Z}_{\mathrm{UV}}$ transforms by a $\mathbb{Z}_{2 N_{c} N_{f}}$ phase for general values of the background BCF fluxes.

\footnotetext{
${ }^{12}$ We stress that while comparing the results in these references to the ones given here, one should keep in mind that $N_{f}$ in this paper denotes the number of Dirac, not Weyl flavors. Thus, the discussion here applies to even numbers of Weyl flavors.

${ }^{13}$ The $\mathrm{U}(1)_{B}$ background is taken to have an extra flux $m^{B} \in \mathbb{Z}, F^{B}=\left(\frac{1}{2}+m^{B}+\frac{n^{f}}{N_{f}} m^{f}\right) K$, cf. (2.7).
} 
Now, we first examine the IR scenario [30] for $N_{c}=2$ and a single Dirac fermion $N_{f}=$ 1. The IR composite Dirac fermion has unit charge under $\mathrm{U}(1)_{B}$ and charge 3 under the $\mathbb{Z}_{8}$ discrete chiral symmetry. ${ }^{14}$ The Dirac index in the IR is obtained by setting $Q^{c}=Q^{f}=0$ in $(2.11)$, which gives $\mathcal{J}_{D}=\frac{1}{2} m^{B}\left(m^{B}+1\right)$. Thus, we find $\left(\left.\mathcal{Z}_{\mathrm{IR}}\right|_{\mathbb{Z}_{8}}\right)_{\mathbb{C P}^{2}} \rightarrow e^{i \frac{2 \pi \times 3}{8} m^{B}\left(m^{B}+1\right)}$, and hence, from (3.13) we find the ratio

$$
\left(\frac{\left.\mathcal{Z}_{\mathrm{UV}}\right|_{\mathbb{Z}_{8}}}{\mathcal{Z}_{\mathrm{IR}}{\mid \mathbb{Z}_{8}}_{\mathbb{C P}^{2}}}=e^{i \frac{2 \pi}{4}\left(m^{c}\right)^{2}}\right.
$$

We note that on a non-spin manifold, this is a $\mathbb{Z}_{4}$ phase, while it is a $\mathbb{Z}_{2}$ phase on a spin manifold. On $\mathbb{T}^{4}$, the computation follows the same steps, taking $\mathrm{SU}\left(N_{c}\right)$ 't Hooft fluxes (see footnote 4), with $Q^{c}=\frac{m m^{\prime}}{2}, Q^{f}=0$, and taking $Q^{B}=m_{b}\left(m, m^{\prime}, m_{b} \in \mathbb{Z}\right.$ ), we have

$$
\left(\frac{\left.\mathcal{Z}_{\mathrm{UV}}\right|_{\mathbb{Z}_{8}}}{\left.\mathcal{Z}_{\mathrm{IR}}\right|_{\mathbb{Z}_{8}}}\right)_{\mathbb{T}^{4}}=\frac{e^{i \frac{2 \pi}{4}\left(4 \frac{m m^{\prime}}{2}+3 m_{b}\right)}}{e^{i \frac{2 \pi}{4} 3 m_{b}}}=e^{i \frac{2 \pi}{2} m m^{\prime}}
$$

The fact that the UV and IR partition functions with massless composite fermions transform differently under $\mathbb{Z}_{8}$ means that the massless composites cannot be all there is in the IR. In particular, as (3.14), (3.15) show, there is a mixed anomaly between the discrete chiral and center symmetries (the 't Hooft fluxes $m, m^{\prime}, m^{c}$ ) which cannot be matched by the IR fermions. This was already recognized in [30], where it was proposed that there is spontaneous breaking of the chiral symmetry, $\mathbb{Z}_{8} \rightarrow \mathbb{Z}_{4}$, by a four-fermion condensate $\left\langle\operatorname{det} \lambda^{i} \lambda^{j}\right\rangle,{ }^{15}$ and that domain walls, via a TQFT coupled to the background fields and describing the two $\mathbb{Z}_{8} \rightarrow \mathbb{Z}_{4}$ vacua, match the mixed discrete-chiral center anomaly.

Consider, however, a chiral transformation in the unbroken $\mathbb{Z}_{4}$. A look at (3.14) and (3.15) shows that an unbroken- $\mathbb{Z}_{4}$ transformation (a $\mathbb{Z}_{8}$ transformation applied twice) generates no phase on $\mathbb{T}^{4}$, but does give rise to a $\mathbb{Z}_{2}$ phase on $\mathbb{C P}^{2}$. The DW theory, however, is blind ${ }^{16}$ to the unbroken $\mathbb{Z}_{4}$ group and only matches the anomalies for the broken symmetries, generated by odd powers of $e^{i \frac{2 \pi}{8}}$. Thus to match the anomaly of the unbroken $\mathbb{Z}_{4}$ group [23], the scenario proposed in [30] has to be modified. The need for such modification is only visible — as (3.14), (3.15) show — when the theory is placed in consistent non-spin backgrounds. It was argued that one would need to supplement the IR with an extra emergent TQFT and an explicit construction of this TQFT as an emergent $\mathbb{Z}_{2}$ gauge theory matching the anomaly of the unbroken $\mathbb{Z}_{4}$ on non-spin manifolds (giving rise to the $\mathbb{Z}_{2}$ phase) was given [16, 19, 23, 31].

Next, we examine the scenario of [32]. The massless composites (3.11) have unit $\mathrm{U}(1)_{B}$ and $\mathbb{Z}_{4 N_{c} N_{f}}$ charges, hence the index in the IR is $\mathcal{J}_{D}=\left(N_{c}^{2}-1\right)\left[Q^{f}+N_{F}\left(Q^{B}+Q^{G}\right)\right]=$

\footnotetext{
${ }^{14}$ Recall that $\mathrm{U}(1)_{B}$ is really the third component of the enhanced $\mathrm{SU}(2)$ flavor symmetry of the two-Weyl theory and that the massless fermion is an $\mathrm{SU}(2)$ doublet.

${ }^{15}$ The determinant is taken in the 2-dimensional space of Weyl flavors.

${ }^{16} \mathrm{~A}$ theory with two vacua and domain walls between should be described, in the IR, by a $\mathbb{Z}_{2}$ TQFT with Euclidean Lagrangian $i \frac{2}{2 \pi} \int \phi^{(0)}\left(d a^{(3)}+\ldots\right)$, see [48] for a recent discussion. Here, $\phi^{(0)}$ and $a^{(3)}$ are compact 0 -form and 3-form gauge fields $\left(d \phi^{(0)}\right.$ and $d a^{(3)}$ have periods $2 \pi \mathbb{Z}$ when integrated over appropriate cycles) and the dots denote background field couplings. Under the action of the broken $\mathbb{Z}_{8}$ generators, $\phi^{(0)}$ shifts by $\pi$, but is inert under the unbroken $\mathbb{Z}_{4}$ generators.
} 
$\left(N_{c}^{2}-1\right) \frac{m_{f}\left(m_{f}+1\right)}{2}$. Thus, we find, proceeding as above and taking $m^{B}=0$ with no loss of generality, that

$$
\left(\frac{\left.\mathcal{Z}_{\mathrm{UV}}\right|_{\mathbb{Z}_{4 N_{f} N_{c}}}}{\left.\mathcal{Z}_{\mathrm{IR}}\right|_{\mathbb{Z}_{4 N_{f} N_{c}}}}\right)_{\mathbb{C P}^{2}}=e^{i \frac{2 \pi\left(m^{c}\right)^{2}}{2}\left(1-\frac{1}{N_{c}}\right)} .
$$

Again, we find that this phase is half the phase one obtains from the mixed anomaly between the discrete chiral and center symmetries on spin manifolds. Ref. [32] proposed that a higher-dimensional condensate breaks $\mathbb{Z}_{4 N_{f} N_{c}} \rightarrow \mathbb{Z}_{4 N_{f}}$, but as in the above $N_{f}=1, N_{c}=2$ example, this is not sufficient to match the anomaly of the unbroken $\mathbb{Z}_{4 N_{f}}$ symmetry on $\mathbb{C P}^{2}$ (it is clear, by applying (3.16) $N_{c}$ times, that this is a $\mathbb{Z}_{2}$-valued anomaly). Thus, we conclude that an additional emergent TQFT, argued to also be an emergent $\mathbb{Z}_{2}$ gauge theory [16], has to exist in the IR to match the anomaly of the unbroken $\mathbb{Z}_{4 N_{f}}$ symmetry on $\mathbb{C P}^{2}$.

To summarize, in both scenarios [30, 32], the IR theory consists of three decoupled sectors: massless composite fermions, a $\mathbb{Z}_{N_{c}}$ TQFT due to the spontaneous chiral symmetry breaking (with $N_{c}$ vacua and domain walls), and an emergent topological $\mathbb{Z}_{2}$ gauge theory. Here, we shall not speculate on the likelihood of this scenario and simply refer the reader to [47] for the up-to-date status of the lattice studies.

\subsection{2 $\mathrm{SU}(6)$ with a Dirac fermion in the two-index anti-symmetric represen- tation}

As our second study of the new anomaly, we consider $\mathrm{SU}\left(N_{c}=6\right)$ vector-like theory with a single Dirac spinor with $\mathcal{R}$ taken to be the two-index antisymmetric representation $(N$-ality $n_{c}=2$ ). We denote its two undotted Weyl-fermion components as $\psi, \tilde{\psi}$, transforming in $\mathcal{R}$ and $\overline{\mathcal{R}}$, respectively. Recalling (2.1), the global symmetry of this theory is

$$
G^{\text {global }}=\frac{U_{B}(1) \times \mathbb{Z}_{8}}{\mathbb{Z}_{3} \times \mathbb{Z}_{2}} \times \mathbb{Z}_{2}^{(1)},
$$

where we modded by the $\mathbb{Z}_{3}$, the discrete group that acts faithfully on fermions, and the $\mathbb{Z}_{2}$ subgroup of the Lorentz group, while the 1 -form center symmetry $\mathbb{Z}_{2}^{(1)}$ should be understood as acting on topologically nontrivial Wilson loops.

A possible phase of the theory is one where a bilinear fermion condensate $\langle\tilde{\psi} \psi\rangle$ forms. This condensate preserves the vectorlike $\mathrm{U}(1)_{B}$ but breaks $\mathbb{Z}_{8}$ down to $\mathbb{Z}_{2}$. The theory is gapped and in the deep IR the anomaly is matched by a $\mathbb{Z}_{4}$ TQFT describing the four ground states of the theory. This number of vacua is consistent with the constraints on gapped phases of such theories recently derived in [19]. This is also the breaking pattern expected when the theory is coupled to an axion [22].

In what follows, we study the viability of a more exotic scenario for the IR physics, namely the possibility to match the anomalies via a single massless composite Dirac fermion of the form ${ }^{17} \mathcal{O} \sim(\psi)^{3}, \tilde{\mathcal{O}} \sim(\tilde{\psi})^{3}$, which has charge 3 under both $\mathrm{U}(1)_{B}$ and $\mathbb{Z}_{8}$. It is a

\footnotetext{
${ }^{17}$ Derivative and field strength insertions may be required in the precise definition of $\mathcal{O}, \tilde{\mathcal{O}}$. These, however, do not affect the $\mathrm{U}(1)_{B}$ and $\mathbb{Z}_{8}$ quantum numbers of relevance here.
} 
simple exercise to check that all the 0 -form anomalies are matched by the $\mathcal{O}$ composite. Using (i) in (3.7), ignoring (ii), (iii), and (iv) since we are dealing with a single Dirac fermion, one can easily show that there is no integer $\ell^{c}$ that satisfies (3.7). Hence, additional IR data to a massless fermions spectrum is needed.

Next, we check whether $\mathcal{O}$ matches the BCF 't Hooft anomaly on $\mathbb{C P}^{2}$. We will also compare the result with that of the BCF anomaly on a spin manifold. To this end, let us examine the change of the partition function under a global $\mathbb{Z}_{8}$ chiral transformation in the background of the BC fluxes on $\mathbb{C P}^{2}$. From (3.1), using (2.9), (2.10) with $m_{f}=0$, and recalling that the anomaly is twice the Dirac index $(2.11), \mathcal{J}_{D}=\frac{5}{2} m^{c}\left(m^{c}+1\right)$, we find in the UV:

$$
\left(\left.\mathcal{Z}_{\mathrm{UV}}\right|_{\mathbb{Z}_{8}}\right)_{\mathbb{C P}^{2}} \rightarrow \mathcal{Z} e^{i \frac{2 \pi}{8} 2 \mathcal{J}_{D}}=\mathcal{Z} e^{i 2 \pi \frac{m^{c}\left(m^{c}+1\right)}{8}}
$$

In the IR, the Dirac index ${ }^{18}$ for the composite $\mathcal{O}$ is $\mathcal{J}_{D}=1+\frac{m^{c}}{2}\left(m^{c}+3\right)$, thus we find

$$
\left(\left.\mathcal{Z}_{\mathrm{IR}}\right|_{\mathbb{Z}_{8}}\right)_{\mathbb{C P}^{2}} \rightarrow \mathcal{Z} e^{i \frac{2 \pi}{8} 3 \times 2 \mathcal{J}_{D}}=\mathcal{Z} e^{i 2 \pi \frac{3}{4}\left(1+\frac{m^{c}\left(m^{c}+3\right)}{2}\right)}
$$

Therefore, the ratio between the $\mathbb{Z}_{8}$ chiral transformations of the partition function in the UV and IR theories in the same BC background (2.7) is

$$
\left(\frac{\left.\mathcal{Z}_{\mathrm{UV}}\right|_{\mathbb{Z}_{8}}}{\left.\mathcal{Z}_{\mathrm{IR}}\right|_{\mathbb{Z}_{8}}}\right)_{\mathbb{C P}^{2}}=e^{i \frac{2 \pi}{4}\left(-1+\left(m^{c}\right)^{2}\right)}
$$

If the massless composite $\mathcal{O}$ matches all anomalies, the phase on the r.h.s. of (3.20) should be unity for all values of the $\mathrm{SU}\left(N_{c}\right)$ 't Hooft fluxes $m^{c}$. Clearly, this is not the case and (3.20) implies that there is a $\frac{\pi}{2}$ phase mismatch between the UV and IR 't Hooft anomalies on $\mathbb{C P}^{2}$. This phase is obtained even if we completely turn off the $\mathrm{SU}\left(N_{c}\right)$ 't Hooft fluxes by setting $m^{c}=0$, hence the anomaly is solely due to putting the theory on a non-spin manifold, i.e., there is a mixed anomaly between the 0 -form $\mathbb{Z}_{8}$ discrete chiral symmetry and the $\mathrm{U}(1)_{B}$ - gravity background required to put the theory on $\mathbb{C P}^{2}$. The mismatch (3.20) indicates that a single composite in the IR cannot by itself match this mixed anomaly. In addition to the composite, the theory has to be supplemented by partial breaking of $\mathbb{Z}_{8}$ and/or an IR TQFT.

It is also important to compare the situation with the $\mathrm{BCF}$ anomaly on a spin manifold. One can repeat the above exercise on $\mathbb{T}^{4}$ to find, in the background of $\mathrm{BC}$ fluxes (recall (3.15))

$$
\left(\frac{\left.\mathcal{Z}_{\mathrm{UV}}\right|_{\mathbb{Z}_{8}}}{\left.\mathcal{Z}_{\mathrm{IR}}\right|_{\mathbb{Z}_{8}}}\right)_{\mathbb{T}^{4}}=e^{i \pi m m^{\prime}}
$$

instead of (3.20) on $\mathbb{C P}^{2}$. The $\pi$ phase mismatch can also be obtained as the result of a mixed anomaly between $\mathbb{Z}_{8}$ and the 1 -form $\mathbb{Z}_{2}^{(1)}$ center symmetry [15]. In both $\mathbb{C P}^{2}$ and $\mathbb{T}^{4}$ cases (3.20), (3.21) we find that one needs to supplement the theory with an emergent IR

\footnotetext{
${ }^{18}$ The IR composite only couples to the gravitational and baryon number backgrounds (2.7), hence $Q^{c}=Q^{f}=0$. In addition, since the baryon charge of $\mathcal{O}$ is 3 , the formula for the index (2.11) has to be modified by multiplying $Q_{B}$ by $3^{2}$ and taking $\operatorname{dim}_{\mathcal{R}^{f}} \operatorname{dim}_{\mathcal{R}^{c}}=1$.
} 
TQFT in order to match the phases in (3.20), (3.21). The $\mathbb{T}^{4} \mathrm{UV} / \mathrm{IR}$ phase mismatch (3.21), for the broken $\mathbb{Z}_{8}$ generators, could be due to domain walls from the spontaneous breaking $\mathbb{Z}_{8} \rightarrow \mathbb{Z}_{4}$ by a $(\tilde{\psi} \psi)^{2}$-condensate (recall also that $\mathbb{Z}_{2} \in \mathbb{Z}_{4}$ is fermion number). This, however would not match the nontrivial $\mathbb{Z}_{2}$-valued anomaly in the unbroken- $\mathbb{Z}_{4}$ transformation of the partition function on a non-spin manifold (3.20). Thus, we conclude that, once again, putting the theory on a non-spin manifold gives more constraints on the IR physics, by requiring an extra TQFT to match the anomaly of the unbroken $\mathbb{Z}_{4}$ symmetry on $\mathbb{C P}^{2}$ (the phase to be matched is, again, a $\mathbb{Z}_{2}$ phase). The results of [16] imply that such a $\mathbb{Z}_{4}$ and $\mathbb{Z}_{2}^{(1)}$-center symmetric TQFT exists: the anomaly inflow action is nontrivial if one assumes $\mathbb{Z}_{8}$ and $\mathbb{Z}_{2}^{(1)}$ unbroken symmetries (precluding the existence of a symmetric gapped phase [16]), but trivializes for the case of unbroken $\mathbb{Z}_{4}$ and $\mathbb{Z}_{2}^{(1)}$. See also the discussion of the more general case near eq. (3.30) in the following section.

\subsubsection{SU $(4 k+2)$ with fermions in the two-index (anti)-symmetric represen- tation}

Here, we generalize the $\mathrm{SU}(6)$-theory analysis to $\mathrm{SU}(4 k+2)$ with a single Dirac fermion in the two-index symmetric (S) or anti-symmetric (AS) representation. ${ }^{19}$ The conclusion, with regards to an IR phase with composite massless fermions, is essentially the same as in the $\mathrm{SU}(6)$ theory of section 3.1.2. Below, we give the details for completeness.

We turn on color and baryon-number fluxes and use (2.11) to calculate the Dirac index in the UV. Recalling that $Q^{c}=\frac{\left(m^{c}\right)^{2}}{2}\left(1-\frac{1}{4 k+2}\right), Q^{B}=\frac{1}{2}\left(\frac{1}{2}+\frac{2 m^{c}}{4 k+2}\right)^{2}, T_{\mathrm{S}, \mathrm{AS}}=4 k+2 \pm 2$, and $\left.\operatorname{dim}_{\mathrm{S}, \mathrm{AS}}=\frac{1}{2}(4 k+2)(4 k+2 \pm 1)\right)$, we find

$$
\mathcal{J}_{D}^{\mathrm{UV}}= \begin{cases}\frac{m^{c}}{2}\left(3+5 m^{c}+4 k\left(1+m^{c}\right)\right) & \mathrm{S}, \\ \frac{m^{c}}{2}\left(m^{c}+1\right)(4 k+1) & \mathrm{AS},\end{cases}
$$

from which one can readily find that the partition function receives the following phases upon performing a discrete chiral symmetry transformation $\mathbb{Z}_{2(4 k+2 \pm 2)}$ :

$$
\left(\left.\mathcal{Z}_{\mathrm{UV}}\right|_{\mathbb{Z}_{2(4 k+2 \pm 2)}}\right)_{\mathbb{C P}^{2}} \rightarrow \mathcal{Z} e^{i \frac{2 \pi}{4 k+2 \pm 2}} \mathcal{J}_{D}^{\mathrm{UV}}
$$

As above, we focus on the anomaly constraints on an exotic scenario for the IR physics. We assume that the 0-form anomalies are saturated in the IR by a set of massless composites. This can be achieved in the AS case by a single composite $\mathcal{O} \sim(\psi)^{2 k+1}$ and single anti-composite $\tilde{\mathcal{O}} \sim(\tilde{\psi})^{2 k+1}$, while in the $\mathrm{S}$ case we need ${ }^{20} 3+4 k$ composites $\mathcal{O} \sim(\psi)^{2 k+1}$ and anti-composites $\tilde{\mathcal{O}} \sim(\tilde{\psi})^{2 k+1}$, possibly with appropriate insertions of derivatives and/or gluonic fields. Since all the IR composites are color singlets, only the baryon flux will contribute to the Dirac index:

$$
\mathcal{J}_{D}^{\mathrm{IR}}=(2 k+1)^{2} Q^{B}-\frac{1}{8}=\frac{1}{2}\left[k(k+1)+m^{c}\left(1+2 k+m^{c}\right)\right]
$$

\footnotetext{
${ }^{19}$ Notice that SU(4k) with fermions in the two-index S or AS does not admit color-singlet fermions in the IR. Hence, we exclude this case from our discussion.

${ }^{20}$ To match the 0 -form anomalies involving $\mathbb{Z}_{2(4 k+4)}$.
} 
for each of the symmetric and anti-symmetric Dirac composites, and we used the fact that the $\mathrm{U}(1)_{B}$ charges of the composites is $2 k+1$. Using this information, we obtain the following phases in the partition function upon performing a discrete chiral transformation:

$$
\left(\left.\mathcal{Z}_{\mathrm{IR}}\right|_{\mathbb{Z}_{2(4 k+2 \pm 2)}}\right)_{\mathbb{C P}^{2}} \rightarrow \mathcal{Z} \times\left\{\begin{array}{ll}
e^{i 2 \pi \frac{(2 k+1)(3+4 k) \mathcal{J}_{D}^{\mathrm{IR}}}{4 k+4}} & \mathrm{~S} \\
e^{i 2 \pi \frac{(2 k+1) \mathcal{J}_{D}^{\mathrm{IR}}}{4 k}} & \mathrm{AS}
\end{array},\right.
$$

where we used the fact that we need $3+4 k$ composites in the symmetric case. Finally, after some algebra we obtain the ratios:

$$
\left(\frac{\left.\mathcal{Z}_{\mathrm{UV}}\right|_{\mathbb{Z}_{2(4 k+2 \pm 2)}}}{\left.\mathcal{Z}_{\mathrm{IR}}\right|_{\mathbb{Z}_{2(4 k+2 \pm 2)}}}\right)_{\mathbb{C P}^{2}}= \begin{cases}e^{i 2 \pi \frac{-3 k-10 k^{2}-12 k m^{c}+2\left(m^{c}\right)^{2}}{8}} & \mathrm{~S} \\ e^{i 2 \pi \frac{-1-2 k^{2}+2\left(m^{c}\right)^{2}-k\left(3+4 m^{c}\right)}{8}} & \mathrm{AS}\end{cases}
$$

This phase mismatch between the UV and IR implies that turning on BC fluxes on $\mathbb{C P}^{2}$ rules out the set of composites as the sole spectrum in the IR. For the S case, we obtain a $\mathbb{Z}_{8}$-valued anomaly on $\mathbb{C P}^{2}$ for odd values of $k$, and a $\mathbb{Z}_{4}$-valued one for even values of $k$, while for the AS case we obtain a $\mathbb{Z}_{4}$ phase for odd- $k$ and a $\mathbb{Z}_{8}$ phase for even- $k$.

Before we continue with studying the implications of (3.26), let us contrast the situation on $\mathbb{C P}^{2}$ with that on $\mathbb{T}^{4}$. In the latter case we can turn on general color and baryon fluxes in the 1-2 and 3-4 planes: $Q^{c}=m_{12}^{c} m_{34}^{c}\left(1-\frac{1}{4 k+2}\right), Q^{B}=\frac{4 m_{12}^{c} m_{34}^{c}}{(4 k+2)^{2}}$. Then, the Dirac index in the UV is given by

$$
\mathcal{J}_{D}^{\mathrm{UV}}=m_{12}^{c} m_{34}^{c}(4 k+3 \pm 2),
$$

for the S and AS cases, respectively. In the IR the composites are color singlets, they have charge $2 k+1$ under $\mathrm{U}(1)_{B}$, and therefore, the index is

$$
\mathcal{J}_{D}^{\mathrm{IR}}=(2 k+1)^{2} \frac{4 m_{12}^{c} m_{34}^{c}}{(4 k+2)^{2}}=m_{12}^{c} m_{34}^{c} .
$$

Repeating the above steps, we obtain the following phases upon performing a $\mathbb{Z}_{2(4 k+2 \pm 2)}$ discrete chiral transformations in the BC fluxes:

$$
\left(\frac{\left.\mathcal{Z}_{\mathrm{UV}}\right|_{\mathbb{Z}_{2(4 k+2 \pm 2)}}}{\left.\mathcal{Z}_{\mathrm{IR}}\right|_{\mathbb{Z}_{2(4 k+2 \pm 2)}}}\right)_{\mathbb{T}^{4}}=\left\{\begin{array}{ll}
e^{i \pi m_{12}^{c} m_{34}^{c}} & \mathrm{~S} \\
e^{i \pi m_{12}^{c} m_{34}^{c}} & \mathrm{AS}
\end{array} .\right.
$$

Here, the phase we obtain is the exact same $\mathbb{Z}_{2}$ phase one encounters from the discretechiral/1-form $\mathbb{Z}_{2}$-center anomaly.

The symmetry breaking scenario consistent with the above massless composite spectrum is as follows. For the case of symmetric tensor (S) representation, we assume a nonvanishing $(\psi \tilde{\psi})^{2 k+2}$ condensate (with all other condensates zero) breaking the chiral symmetry $\mathbb{Z}_{2(4 k+4)} \rightarrow \mathbb{Z}_{4 k+4}$. The anomaly inflow 5 d action has the form

$$
e^{i \frac{2 \pi}{2} \int_{M_{5}} \frac{2(4 k+4) A^{(1)}}{2 \pi} \wedge \frac{2 B^{(2)}}{2 \pi} \wedge \frac{2 B^{(2)}}{2 \pi},}
$$

with $A^{(1)}$ a 1-form gauge field for $\mathbb{Z}_{2(4 k+4)}$ and $B^{(2)}$ a 2 -form gauge field for the $\mathbb{Z}_{2}^{(1)}$ center symmetry. ${ }^{21}$ The chiral variation of (3.30) reproduces the $\mathbb{Z}_{2}$-valued mixed anomaly (3.29).

\footnotetext{
${ }^{21}$ The normalization and transformation properties of $A^{(1)}$ and $B^{(2)}$ are as in [4-6].
} 
In addition, (3.30) evaluates to $e^{i \pi}$ on $\mathbb{S}^{1} \times \mathbb{S}^{2} \times \mathbb{S}^{2}$, thus, according to [16] no $\mathbb{Z}_{2(4 k+4)^{-}}$and $\mathbb{Z}_{2}^{(1)}$-symmetric unitary TQFT exists to match this anomaly, implying that the symmetry has to suffer at least partial breakdown. However, when $\oint \frac{2(4 k+4) A^{(1)}}{2 \pi}=2$, i.e. with the background restricted to the unbroken $\mathbb{Z}_{4 k+4}$, the expression (3.30) evaluates to unity and a symmetric TQFT matching the unbroken symmetries anomaly is not excluded. ${ }^{22}$ A similar scenario with $\mathbb{Z}_{8 k} \rightarrow \mathbb{Z}_{4 k}$ symmetry breaking, due to a nonzero $(\psi \tilde{\psi})^{2 k}$ condensate, holds for the AS case.

As in the composite-fermion $\mathrm{QCD}(\mathrm{adj})$ scenarios discussed in the previous section, there are three decoupled sectors in the IR: massless composite fermions, domain walls and multiple vacua due to the symmetry breaking, and a TQFT to match the anomaly of the unbroken chiral symmetry. As before, we shall not dwell on the likelihood of these exotic IR phases appearing in the nonabelian gauge theories under consideration.

\subsection{Comments on future studies}

In this section, we studied a few examples illustrating the utility of the mixed chiral/BCF anomaly on non-spin backgrounds. Our main focus was on exotic phases where massless composite fermions saturate the "traditional" 0-form 't Hooft anomalies. The main lesson we take is that the new generalized 't Hooft anomalies on both spin and non-spin manifolds yield further constraints.

It is clear that generalized 't Hooft anomalies will also have implications on the physics of "vanilla" phases where fermion bilinears obtain expectation values maximally breaking the chiral symmetries. As the analysis [23] of SU(2) QCD(adj) with a single Dirac flavor showed, the structure of the IR theory, its domain walls, and confining strings can reflect the anomalies in an intricate way. It would be interesting to understand the implications of anomaly matching for similar phases in more general theories, including chiral theories or the ones studied in [14]. Constructing the IR TQFTs that must accompany the various exotic phases mentioned here is also of interest (we also note that their UV origin remains mysterious). Anomalies should also have implications for the finite temperature phase structure, as in $[5,7,50-52]$.

\section{Acknowledgments}

We thank Georg Bergner and Andreas Wipf for many interesting discussions. MA gratefully acknowledges the hospitality at the University of Jena and the University of Toronto. MA is supported by the NSF grant PHY-1720135. EP is supported by a Discovery Grant from NSERC.

\section{A Some useful formulae for $\mathbb{C P}^{2}$}

In this appendix, we review important facts about the complex projective space $\mathbb{C P}^{2}$. Our notation largely follows $[53,54] . \mathbb{C P}^{2}$ is the set of lines in the three-dimensional complex

\footnotetext{
${ }^{22}$ The recent ref. [49] asserts that all symmetric TQFTs not excluded by [16] do in fact exist.
} 
space, $\mathbb{C}^{3}$, passing through the origin. $\mathbb{C P}^{2}$ can be described by the complex coordinates $\Xi=\left(\xi_{1}, \xi_{2}, \xi_{3}\right) \neq(0,0,0)$ (here $\left.\xi_{1,2,3} \in \mathbb{C}\right)$ modulo the identification $\Xi \equiv \lambda \Xi$ for any complex number $\lambda \neq 0$. One can cover $\mathbb{C P}^{2}$ with three patches $U_{i}\left(i=1,2,3\right.$, where $U_{i}$ covers $\xi_{i} \neq 0$ ) such that the transition functions on the overlap $U_{i} \cap U_{j}$ are holomorphic. $\mathbb{C P}^{2}$ is a Kähler manifold, with a Kähler 2-form given by

$$
K=i \partial \wedge \bar{\partial} \mathcal{K}
$$

where $\partial$ is defined as $\partial f \equiv \sum_{\alpha} \frac{\partial f}{\partial z^{\alpha}} d z^{\alpha}$ (and similarly for $\bar{\partial}$ ) and $\mathcal{K}$ is the Kähler potential:

$$
\mathcal{K}=\log \left(1+\sum_{\alpha=1}^{2} z^{\alpha} \bar{z}^{\alpha}\right),
$$

where $z^{1,2}$ cover one of the patches $U_{i}$. Taking $z^{1} \equiv \xi^{1} / \xi^{3}, z^{2} \equiv \xi^{2} / \xi^{3}$, this is the $U_{3}$ patch with $\xi_{3} \neq 0$. At the points $\xi^{3}=0$ in $\mathbb{C P}^{2}$, we have $\left(\xi^{1}, \xi^{2}\right) \equiv \lambda\left(\xi^{1}, \xi^{2}\right)$, i.e. a two-sphere $\mathbb{S}^{2}=\mathbb{C P}^{1}$. In the coordinates used in (A.2), the $\mathbb{S}^{2}$ is at $|z|^{\alpha} \rightarrow \infty$. (This is also clear from the explicit expression for the metric (A.5), shown in polar coordinates in (A.10).)

The Kähler 2-form (A.1) is closed, $d K=0$, and co-closed, $\delta K=0$, and is associated to the metric tensor $g_{\alpha \bar{\beta}}$ :

$$
K=i g_{\alpha \bar{\beta}} d z^{\alpha} \wedge d \bar{z}^{\beta}
$$

Therefore, we immediately find

$$
g_{\alpha \bar{\beta}}=\frac{\delta_{\alpha \beta}}{1+\sum_{\alpha=1}^{2} z^{\alpha} \bar{z}^{\alpha}}-\frac{\bar{z}^{\alpha} z^{\beta}}{\left(1+\sum_{\alpha=1}^{2} z^{\alpha} \bar{z}^{\alpha}\right)^{2}} .
$$

Now, one can set $z^{1}=x+i y$ and $z^{2}=z+i t$ to find that the metric on $\mathbb{C P}^{2}$ can be written in the Fubini-Study form:

$$
d s^{2}=g_{\alpha \bar{\beta}} d z^{\alpha} d \bar{z}^{\beta}=\frac{d r^{2}+r^{2} \sigma_{z}^{2}}{\left(1+r^{2}\right)^{2}}+\frac{r^{2}\left(\sigma_{x}^{2}+\sigma_{y}^{2}\right)}{1+r^{2}},
$$

where $r^{2}=x^{2}+y^{2}+z^{2}+t^{2}$ and $\sigma_{x, y, z}$ are the left-invariant 1-forms on the manifold of the group $\mathrm{SU}(2)=\mathbb{S}^{3}$, obeying $d \sigma_{x}=2 \sigma_{y} \wedge \sigma_{z}$ (plus cyclic). The latter are given in terms of the $x, y, z, t$ coordinates by:

$$
\begin{aligned}
\sigma_{x} & =\frac{-t d x-z d y+y d z+x d t}{r^{2}}, \\
\sigma_{z} & =\frac{-y d x+x d y-t d z+z d t}{r^{2}}
\end{aligned}
$$

For our explicit calculations of appendix B, we introduce polar coordinates $r, \theta, \phi, \psi$

$$
z_{1}=x+i y=r \cos \frac{\theta}{2} e^{i \frac{\psi+\phi}{2}}, \quad z_{2}=z+i t=r \sin \frac{\theta}{2} e^{i \frac{\psi-\phi}{2}},
$$


where $0 \leq r<\infty, 0 \leq \theta<\pi, 0 \leq \phi<2 \pi, 0 \leq \psi<4 \pi$. The 1 -forms $\sigma_{x, y, z}$ are now

$$
\begin{aligned}
\sigma_{x} & =\frac{-\cos \psi \sin \theta d \phi+\sin \psi d \theta}{2}, \quad \sigma_{y}=-\frac{\cos \psi d \theta+\sin \theta \sin \psi d \phi}{2}, \\
\sigma_{z} & =\frac{d \psi+\cos \theta d \phi}{2}
\end{aligned}
$$

One also can write the metric in terms of the vierbein 1-forms as $d s^{2}=e^{a} e^{b} \eta_{a b}$, where $\eta_{a b}$ is the flat Euclidean metric. Then, by inspecting (A.5) one immediately finds:

$$
e^{0}=\frac{d r}{1+r^{2}}, \quad e^{1}=\frac{r \sigma_{x}}{\sqrt{1+r^{2}}}, \quad e^{2}=\frac{r \sigma_{y}}{\sqrt{1+r^{2}}}, \quad e^{3}=\frac{r \sigma_{z}}{1+r^{2}} .
$$

In terms of the vierbein (A.8), the Kähler 2-form (A.1) is

$$
K=2\left(e^{0} \wedge e^{3}+e^{1} \wedge e^{2}\right)=\frac{r}{\left(1+r^{2}\right)^{2}} d r \wedge(d \psi+\cos \theta d \phi)-\frac{1}{2} \frac{r^{2}}{1+r^{2}} \sin \theta d \theta \wedge d \phi,
$$

from which one can see that $K$ is anti-self-dual $\star K=-K\left(\epsilon_{1230}=1\right)$. We use the Kähler form $K$ in polar coordinates in the calculations of fluxes and topological charges in appendix B. In particular, note that $\int_{\mathbb{C P}^{2}} K \wedge K=\frac{8 \pi^{2}}{2}$.

The Fubini-Study metric (A.5), explicitly written using polar coordinates (A.6), is

$$
d s^{2}=\frac{d r^{2}}{\left(1+r^{2}\right)^{2}}+\frac{r^{2}}{4\left(1+r^{2}\right)^{2}}(d \psi+\cos \theta d \phi)^{2}+\frac{r^{2}}{4\left(1+r^{2}\right)}\left(d \theta^{2}+\sin ^{2} \theta d \phi^{2}\right) .
$$

To study the points at $r \rightarrow \infty$, one can introduce a new coordinate $u=1 / r$ and observe that at $u=0$ there is a $\mathbb{S}^{2}$ of area $\pi$ (the metric is well behaved at $u=0$; the singularity apparent in the first two terms of (A.10) at $1 / r=u \rightarrow 0$ is only a coordinate one, see [53]).

The Ricci tensor of the Fubini-Study metric (A.10) is $R_{a b}=6 \delta_{a b}$, so it is a solution of the Einstein's equation $R_{a b}-\frac{1}{2} \delta_{a b} R=-\Lambda \delta_{a b}$ with the energy-momentum tensor being that of a cosmological constant $\Lambda=+6$. This holds for the form of $\mathcal{K}$ given in (A.2), with dimensionless coordinates $z^{\alpha}$. If, instead of (A.2), we take $\mathcal{K}=\frac{6}{\Lambda} \log \left(1+\frac{\Lambda}{6} \sum_{\alpha=1}^{2} z^{\alpha} \bar{z}^{\alpha}\right)$, we shall find $R_{a b}=\Lambda \delta_{a b}$, for arbitrary $\Lambda$.

Thus the compact manifold $\mathbb{C P}^{2}$ has a size scaling as $\Lambda^{-\frac{1}{2}}$. It can be taken to have any size, in particular it can be larger than $\Lambda_{Q C D}^{-1}$, the inverse strong-coupling scale of the gauge theory. Taking $\Lambda \rightarrow 0$ approaches an infinite volume limit. As in the $\mathbb{T}^{4}$ case, this is the limit of interest from the point of view of constraining infinite volume nonperturbative dynamics via anomaly matching.

\section{B Gauge fields and fermions on $\mathbb{C P}^{2}$}

In order to turn on a $\mathrm{U}(1)$ gauge field (which can be embedded into $\mathrm{SU}\left(N_{c}\right)$, see below) of two-form strength $F$ on $\mathbb{C P}^{2}$, one needs to ensure that the field will not backreact on the manifold, and hence, destroy $\mathbb{C P}^{2}$. This can be achieved by demanding that $F$ is an (anti)self-dual 2-form field, since in this case the field has a vanishing energy-momentum 
tensor. ${ }^{23}$ Therefore, the simplest way to find a consistent solution of the Einstein-Maxwell equations on $\mathbb{C P}^{2}$ is by writing $F$ in terms of the Kähler 2-form as $F=C K$ for some constant $C \in \mathbb{R}$. Below, we will see that defining spinors on $\mathbb{C P}^{2}$ demands that $C$ be quantized in half-integer units.

It is well known that fermions are ill-defined on $\mathbb{C P}^{2}$; we say that $\mathbb{C P}^{2}$ is a non-spin manifold. Briefly, ${ }^{24}$ to see that spinor fields $\Psi$ are not globally well defined, one considers a family of closed contours $\gamma(s)$, with $s \in[0,1]$ parameterizing the different contours. This family of contours wraps the $\mathbb{S}^{2}$ in $\mathbb{C P}^{2}$, such that $\gamma(0)$ and $\gamma(1)$ are the trivial contours. Then one considers the parallel transport of tetrads, and the corresponding uplift to spinors, along each contour belonging to this family. The SO(4) holonomies corresponding to parallel transporting tetrads along the family $\gamma(s)$, considered as a function of $s$, form a closed non-contractible loop in $\mathrm{SO}(4)$ (recall that $\gamma(0)$ and $\gamma(1)$ are trivial contours). Correspondingly, the uplift of the $\mathrm{SO}(4)$ holonomies (for the $s=0$ and $s=1$ curves) to its double cover Spin(4), responsible to transporting the spinors, differ by minus sign. Schematically, one obtains

$$
\Psi(s=1)=e^{i \pi} \Psi(s=0),
$$

showing the global inconsistency (recalling that $\gamma(0)$ and $\gamma(1)$ are both the trivial contour) in defining spinors. ${ }^{25}$

One can also see the problem of formulating spinors on $\mathbb{C P}^{2}$ by computing the index of a Dirac spinor on $\mathbb{C P}^{2}$ :

$$
\mathcal{J}_{D}=\frac{1}{192 \pi^{2}} \int_{\mathbb{C P}^{2}} \operatorname{tr}[R \wedge R]=-\frac{1}{8} .
$$

The fractional value $1 / 8$ one obtains for an integer-valued quantity (the Dirac index) is another manifestation of the failure of $\mathbb{C P}^{2}$ to accommodate spinor fields.

One can define spinor fields on $\mathbb{C P}^{2}$ if one turns on a $\mathrm{U}(1)$ gauge bundle that eats up the $i \pi$ phase in (B.1), which renders the spinors well-defined [26]. In this case one finds that the $e^{i \pi}$ factor in (B.1) gets modified to:

$$
e^{i 2 \pi\left(\frac{1}{2}+e \oint_{\mathbb{C P} 1} \frac{F}{2 \pi}\right)}=1,
$$

where $e$ is the $\mathrm{U}(1)$ charge of the fermions and we used Gauss' law. Then the minus sign that arises from parallel transporting the spinors can be cancelled by the minus sign arising from propagating the $\mathrm{U}(1)$ charges. Thus, one can consistently define charged spinors in this $\mathrm{U}(1)$ background. This generalized spin structure is called a $\operatorname{spin}^{c}$ structure.

To obtain the quantization condition on the U(1) flux, we use $F=C K$, as discussed above, along with the expression of the Kähler 2-form in (A.9). We take the limit $r \rightarrow \infty$ and integrate eq. (B.3) over the $\mathbb{S}^{2}$ parametrized by $\theta$ and $\phi$, recall (A.10). We find

\footnotetext{
${ }^{23}$ The kinetic term is $\int_{\mathbb{C P}^{2}} F \wedge \star F$, which, using (anti) self-duality of $F$, becomes $\pm \int_{\mathbb{C P}^{2}} F \wedge F$. The latter is a metric-independent topological term, and hence, its energy-momentum tensor vanishes identically.

${ }^{24}$ For more detail see [24-26].

${ }^{25}$ In a more mathematical language, the second Stiefel-Whitney class of $\mathbb{C P}^{2}$ is non-zero, indicating that there is a sign ambiguity when spinors are parallel-transported around some paths in $\mathbb{C P}^{2}[54]$.
} 
$\oint_{\mathbb{C P}^{1}} K=-2 \pi$, and obtain

$$
1=e^{i 2 \pi\left(\frac{1}{2}+\frac{e C}{2 \pi} \oint_{\mathbb{C P} 1} K\right)}=e^{i 2 \pi\left(\frac{1}{2}-e C\right)} .
$$

Thus, the quantization condition is $e C=m+\frac{1}{2}$ with $m \in \mathbb{Z}$. Without loss of generality we take $e=1$ and conclude that the necessary condition to define spinors on $\mathbb{C P}^{2}$ is to turn on the quantized monopole field

$$
F=\left(m+\frac{1}{2}\right) K
$$

As described in the main text, we also consider turning on the color, flavor, and baryon backgrounds (2.7), reproduced here for convenience

$$
F^{(c)}=\boldsymbol{H}^{c} \cdot \boldsymbol{\nu}^{c} m^{c} K, F^{(f)}=\boldsymbol{H}^{f} \cdot \boldsymbol{\nu}^{f} m^{f} K, F^{B}=\left(\frac{1}{2}+\frac{n^{c}}{N_{c}} m^{c}+\frac{n^{f}}{N_{f}} m^{f}\right) K .
$$

Notice that these are embedded into the Cartan subalgebras of $\mathrm{SU}\left(N_{c}\right)$ and $\mathrm{SU}\left(N_{f}\right)$ and represent a generalization of the BCF 't Hooft flux backgrounds on $\mathbb{T}^{4}$ studied in [20]. When the U(1) background $F=C K$ is replaced by (B.6), we obtain, instead of (B.1), for $\Psi$ of unit charge under baryon number, in a representation of $N_{c}$-ality $n_{c}$ and $N_{f}$-ality $n_{f}$,

$$
\begin{aligned}
\Psi(s=1) & =e^{i 2 \pi\left(\frac{1}{2}+\oint_{\mathbb{C P} 1} \frac{F^{B}+n_{f} F^{(f)}+n_{c} F^{(c)}}{2 \pi}\right)} \Psi(s=0) \\
& =e^{i 2 \pi\left(\frac{1}{2}-\left(\frac{1}{2}+\frac{n^{c}}{N_{c}} m^{c}+\frac{n^{f}}{N_{f}} m^{f}\right)-n_{c} \boldsymbol{H}^{c} \cdot \boldsymbol{\nu}^{c} m^{c}-n_{f} \boldsymbol{H}^{f} \cdot \boldsymbol{\nu}^{f} m^{f}\right)} \Psi(s=0) \\
& =\Psi(s=0),
\end{aligned}
$$

where the last equality follows from the fact that the fractional part of the eigenvalues of $\boldsymbol{H}^{c} \cdot \boldsymbol{\nu}^{c}$ is $-1 / N_{c}$ (and similar for $c \rightarrow f$ ). Thus the background (B.6), or eq. (2.7) of the main text, is consistent with parallel transport on $\mathbb{C P}^{1}$.

The Pontryagin number of the $\mathrm{U}(1)$ bundle, using $\int_{\mathbb{C P}^{2}} K \wedge K=\frac{8 \pi^{2}}{2}$, is given by

$$
\mathcal{P}=\frac{1}{8 \pi^{2}} \int_{\mathbb{C P}^{2}} F \wedge F=\frac{1}{2}\left(m+\frac{1}{2}\right)^{2},
$$

which combines with (B.2) to give the full Dirac index in the combined $\mathrm{U}(1)$ and $\mathbb{C P}^{2}$ background

$$
\mathcal{J}_{D}=\frac{1}{8 \pi^{2}} \int_{\mathbb{C P}^{2}} F \wedge F+\frac{1}{192 \pi^{2}} \int_{\mathbb{C P}^{2}} \operatorname{tr}[R \wedge R]=\frac{m}{2}(m+1) \in \mathbb{Z},
$$

which now has integer values. ${ }^{26}$

Likewise, the Dirac index for the fermions of (B.7), in the background (B.6), is

$$
\mathcal{J}_{D}=T_{\mathcal{R}^{c}} \operatorname{dim}_{\mathcal{R}^{f}} Q^{c}+T_{\mathcal{R}^{f}} \operatorname{dim}_{\mathcal{R}^{c}} Q^{f}+\operatorname{dim}_{\mathcal{R}^{f}} \operatorname{dim}_{\mathcal{R}^{c}}\left(Q^{B}+Q^{G}\right),
$$

\footnotetext{
${ }^{26}$ The zero modes of the Dirac operator on $\mathbb{C P}^{2}$ were studied and explicitly constructed in [55].
} 
also given in (2.11) of the main text, which is also an integer. Here, $Q^{B}=\frac{1}{8 \pi^{2}} \int F^{B} \wedge F^{B}$ and $Q^{c / f}=\frac{1}{8 \pi^{2}} \int \operatorname{tr}\left[F^{(c) /(f)} \wedge F^{(c) /(f)}\right]$, explicitly given by

$$
Q^{c}=\frac{\left(m^{c}\right)^{2}}{2}\left(1-\frac{1}{N_{c}}\right), Q^{f}=\frac{\left(m^{f}\right)^{2}}{2}\left(1-\frac{1}{N_{f}}\right), Q^{B}=\frac{1}{2}\left(\frac{1}{2}+\frac{n^{c}}{N_{c}} m^{c}+\frac{n^{f}}{N_{f}} m^{f}\right)^{2} .
$$

Finally, we note that one can use equations (B.7), (B.10), (B.11) to identify gauge theories that can be consistently formulated on $\mathbb{C P}^{2}$ without turning on global symmetry backgrounds, i.e. by only modifying the conditions on the gauge bundles being summed over in the path integral. Constructions of this type were recently used to uncover a new $\mathrm{SU}(2)$ anomaly [27] on non-spin manifolds (note that in our examples all fermions can be given gauge invariant mass and there is no analogue of the new $\mathrm{SU}(2)$ anomaly).

The simplest such case [28] is that of an $\mathrm{SU}(2)$ theory with $N_{f}$ Dirac fundamental flavors. To see this from our equations, take $N_{c}=2, m^{c}=1, n^{c}=1, Q^{B}=Q^{f}=0$, and check that (B.7) holds and (B.10) is an integer (for any single flavor). This $\mathrm{SU}(2) \mathrm{QCD}(\mathrm{F})$ with $N_{f}$ flavors was interpreted in [28] as emerging near a quantum critical point of a theory of only bosons (heuristically, this is because all gauge invariant operators are bosonic).

Other examples (involving both $\mathrm{SU}(2)$ and other gauge groups) are discussed in [27, 29]. Within the class of theories considered in this paper (specified in section 2.1) the ones that do not require global symmetry backgrounds to be consistently formulated on $\mathbb{C P}^{2}$ must obey

$$
\frac{1}{2}+\frac{n_{c} m^{c}}{N_{c}} \in \mathbb{Z}, \quad T_{\mathcal{R}^{c}} \frac{\left(m^{c}\right)^{2}}{2}\left(1-\frac{1}{N_{c}}\right)-\frac{1}{8} \operatorname{dim}_{\mathcal{R}^{c}} \in \mathbb{Z},
$$

where the second condition, the integrality of the index, should hold once the first is obeyed. We have not exhaustively studied the solutions of the above conditions for general $n_{c}, \mathcal{R}_{c}$ and will only note a few simple cases. The first is $\mathrm{QCD}(\mathrm{F})$ with $N_{f}$ Dirac flavors and an $\mathrm{SU}\left(N_{c}=2 k\right)$ gauge group. As in the $\mathrm{SU}(2)$ theory of [28], it is easy to see that all gauge invariant operators are bosons (or that (B.12) holds). The second set of theories where (B.12) is easily seen to hold is $\mathrm{QCD}(\mathrm{S} / \mathrm{AS})$ with $N_{f} \mathrm{~S} / \mathrm{AS}$ Dirac flavors and an $\mathrm{SU}\left(N_{c}=4 k\right)$ gauge group. As in the other examples, here also all gauge invariants (e.g. baryons and mesons) are bosons.

Open Access. This article is distributed under the terms of the Creative Commons Attribution License (CC-BY 4.0), which permits any use, distribution and reproduction in any medium, provided the original author(s) and source are credited.

\section{References}

[1] G. 't Hooft, Naturalness, chiral symmetry, and spontaneous chiral symmetry breaking, NATO Sci. Ser. B $\mathbf{5 9}$ (1980) 135.

[2] J.L. Rosner, Explorations of compositeness, Comments Mod. Phys. A 1 (1999) 11 [hep-ph/9812537] [INSPIRE]. 
[3] N. Seiberg, Electric-magnetic duality in supersymmetric nonAbelian gauge theories, Nucl. Phys. B 435 (1995) 129 [hep-th/9411149] [INSPIRE].

[4] D. Gaiotto, A. Kapustin, N. Seiberg and B. Willett, Generalized global symmetries, JHEP 02 (2015) 172 [arXiv: 1412.5148] [INSPIRE].

[5] D. Gaiotto, A. Kapustin, Z. Komargodski and N. Seiberg, Theta, time reversal and temperature, JHEP 05 (2017) 091 [arXiv:1703.00501] [INSPIRE].

[6] D. Gaiotto, Z. Komargodski and N. Seiberg, Time-reversal breaking in $Q C D_{4}$, walls and dualities in $2+1$ dimensions, JHEP 01 (2018) 110 [arXiv:1708.06806] [INSPIRE].

[7] Z. Komargodski, T. Sulejmanpasic and M. Ünsal, Walls, anomalies and deconfinement in quantum antiferromagnets, Phys. Rev. B 97 (2018) 054418 [arXiv:1706.05731] [InSPIRE].

[8] T. Sulejmanpasic and Y. Tanizaki, C-P-T anomaly matching in bosonic quantum field theory and spin chains, Phys. Rev. B 97 (2018) 144201 [arXiv:1802.02153] [INSPIRE].

[9] Y. Tanizaki, Anomaly constraint on massless QCD and the role of Skyrmions in chiral symmetry breaking, JHEP 08 (2018) 171 [arXiv: 1807.07666] [INSPIRE].

[10] Y. Tanizaki and T. Sulejmanpasic, Anomaly and global inconsistency matching: $\theta$-angles, $\mathrm{SU}(3) / \mathrm{U}(1)^{2}$ nonlinear $\sigma$-model, $\mathrm{SU}(3)$ chains and its generalizations, Phys. Rev. B 98 (2018) 115126 [arXiv: 1805.11423] [INSPIRE].

[11] Z. Wan and J. Wang, Higher anomalies, higher symmetries and cobordisms I: classification of higher-symmetry-protected topological states and their boundary fermionic/bosonic anomalies via a generalized cobordism theory, Ann. Math. Sci. Appl. 4 (2019) 107 [arXiv: 1812.11967] [INSPIRE].

[12] C. Córdova, D.S. Freed, H.T. Lam and N. Seiberg, Anomalies in the space of coupling constants and their dynamical applications I, SciPost Phys. 8 (2020) 001 [arXiv: 1905.09315] [INSPIRE].

[13] C. Córdova, D.S. Freed, H.T. Lam and N. Seiberg, Anomalies in the space of coupling constants and their dynamical applications II, SciPost Phys. 8 (2020) 002 [arXiv: 1905.13361] [INSPIRE].

[14] M.M. Anber, Self-conjugate QCD, JHEP 10 (2019) 042 [arXiv:1906.10315] [INSPIRE].

[15] S. Bolognesi, K. Konishi and A. Luzio, Gauging 1-form center symmetries in simple $\mathrm{SU}(N)$ gauge theories, JHEP 01 (2020) 048 [arXiv:1909.06598] [INSPIRE].

[16] C. Córdova and K. Ohmori, Anomaly obstructions to symmetry preserving gapped phases, arXiv:1910.04962 [INSPIRE].

[17] Z. Wan, J. Wang and Y. Zheng, Higher anomalies, higher symmetries and cobordisms II: applications to quantum gauge theories, arXiv:1912.13504 [INSPIRE].

[18] Z. Wan and J. Wang, Higher anomalies, higher symmetries and cobordisms III: QCD matter phases anew, arXiv:1912.13514 [INSPIRE].

[19] C. Córdova and K. Ohmori, Anomaly constraints on gapped phases with discrete chiral symmetry, arXiv:1912.13069 [INSPIRE].

[20] M.M. Anber and E. Poppitz, On the baryon-color-flavor (BCF) anomaly in vector-like theories, JHEP 11 (2019) 063 [arXiv: 1909.09027] [INSPIRE].

[21] G. 't Hooft, A property of electric and magnetic flux in nonabelian gauge theories, Nucl. Phys. B 153 (1979) 141 [INSPIRE]. 
[22] M.M. Anber and E. Poppitz, Deconfinement on axion domain walls, JHEP 03 (2020) 124 [arXiv: 2001.03631] [INSPIRE].

[23] C. Córdova and T.T. Dumitrescu, Candidate phases for $\mathrm{SU}(2)$ adjoint $Q C D_{4}$ with two flavors from $\mathcal{N}=2$ supersymmetric Yang-Mills theory, arXiv:1806.09592 [INSPIRE].

[24] R.P. Geroch, Spinor structure of space-times in general relativity. i, J. Math. Phys. 9 (1968) 1739 [INSPIRE].

[25] R.P. Geroch, Spinor structure of space-times in general relativity. II, J. Math. Phys. 11 (1970) 343 [INSPIRE].

[26] S.W. Hawking and C.N. Pope, Generalized spin structures in quantum gravity, Phys. Lett. B 73 (1978) 42.

[27] J. Wang, X.-G. Wen and E. Witten, A new SU(2) anomaly, J. Math. Phys. 60 (2019) 052301 [arXiv: 1810.00844] [INSPIRE].

[28] Z. Bi and T. Senthil, Adventure in topological phase transitions in 3 + 1-D: non-Abelian deconfined quantum criticalities and a possible Duality, Phys. Rev. X 9 (2019) 021034 [arXiv: 1808.07465] [INSPIRE].

[29] J. Davighi and N. Lohitsiri, Anomaly interplay in U(2) gauge theories, arXiv:2001.07731 [INSPIRE].

[30] M.M. Anber and E. Poppitz, Two-flavor adjoint QCD, Phys. Rev. D 98 (2018) 034026 [arXiv: 1805.12290] [INSPIRE].

[31] Z. Wan and J. Wang, Adjoint $Q C D_{4}$, deconfined critical phenomena, symmetry-enriched topological quantum field theory and higher symmetry-extension, Phys. Rev. D 99 (2019) 065013 [arXiv: 1812.11955] [INSPIRE].

[32] E. Poppitz and T.A. Ryttov, Possible new phase for adjoint QCD, Phys. Rev. D 100 (2019) 091901 [arXiv: 1904.11640] [INSPIRE].

[33] S. Raby, S. Dimopoulos and L. Susskind, Tumbling gauge theories, Nucl. Phys. B 169 (1980) 373 [INSPIRE].

[34] Z. Wan and J. Wang, Beyond standard models and grand unifications: anomalies, topological terms and dynamical constraints via cobordisms, arXiv:1910.14668 [INSPIRE].

[35] J. Davighi, B. Gripaios and N. Lohitsiri, Global anomalies in the standard model(s) and beyond, arXiv: 1910.11277 [INSPIRE].

[36] F. Sannino and K. Tuominen, Orientifold theory dynamics and symmetry breaking, Phys. Rev. D 71 (2005) 051901 [hep-ph/0405209] [INSPIRE].

[37] M. Ünsal, Magnetic bion condensation: a new mechanism of confinement and mass gap in four dimensions, Phys. Rev. D 80 (2009) 065001 [arXiv:0709.3269] [INSPIRE].

[38] A. Cherman, M. Shifman and M. Ünsal, Bose-Fermi cancellations without supersymmetry, Phys. Rev. D 99 (2019) 105001 [arXiv: 1812.04642] [INSPIRE].

[39] S. Catterall and F. Sannino, Minimal walking on the lattice, Phys. Rev. D 76 (2007) 034504 [arXiv: 0705.1664] [INSPIRE].

[40] A.J. Hietanen, K. Rummukainen and K. Tuominen, Evolution of the coupling constant in SU(2) lattice gauge theory with two adjoint fermions, Phys. Rev. D 80 (2009) 094504 [arXiv:0904.0864] [INSPIRE]. 
[41] L. Del Debbio et al., The infrared dynamics of minimal walking technicolor, Phys. Rev. D 82 (2010) 014510 [arXiv: 1004.3206] [INSPIRE].

[42] A. Athenodorou, E. Bennett, G. Bergner and B. Lucini, Recent results from SU(2) with one adjoint Dirac fermion, Int. J. Mod. Phys. A 32 (2017) 1747006 [arXiv:1507.08892] [INSPIRE].

[43] G. Bergner et al., Spectrum and mass anomalous dimension of $\mathrm{SU}(2)$ adjoint QCD with two Dirac flavors, Phys. Rev. D 96 (2017) 034504 [arXiv: 1610.01576] [InSPIRE].

[44] G. Bergner et al., Spectrum and mass anomalous dimension of SU(2) gauge theories with fermions in the adjoint representation: from $N_{f}=1 / 2$ to $N_{f}=2$, PoS(LATTICE2016) 237 [arXiv: 1701.08992] [INSPIRE].

[45] G. Bergner et al., Low energy properties of $\mathrm{SU}(2)$ gauge theory with $N_{f}=3 / 2$ flavours of adjoint fermions, JHEP 01 (2018) 119 [arXiv:1712.04692] [INSPIRE].

[46] G. Bergner et al., Indications for infrared conformal behaviour of $\mathrm{SU}(2)$ gauge theory with $N_{f}=3 / 2$ flavours of adjoint fermions, PoS (LATTICE2018) 191 [arXiv: 1811.03847] [INSPIRE].

[47] Z. Bi et al., Lattice analysis of $\mathrm{SU}(2)$ with 1 adjoint dirac flavor, in the proceedings of the $37^{\text {th }}$ International Symposium on Lattice Field Theory (Lattice 2019), June 16-22, Wuhan, Hubei, China (2019), arXiv:1912.11723 [INSPIRE].

[48] Y. Hidaka, M. Nitta and R. Yokokura, Emergent discrete 3-form symmetry and domain walls, Phys. Lett. B 803 (2020) 135290 [arXiv:1912.02782] [InSPIRE].

[49] R. Thorngren, TQFT, symmetry breaking and finite gauge theory in $3+1 D$, arXiv: 2001.11938 [INSPIRE].

[50] H. Shimizu and K. Yonekura, Anomaly constraints on deconfinement and chiral phase transition, Phys. Rev. D 97 (2018) 105011 [arXiv:1706.06104] [INSPIRE].

[51] M.M. Anber and E. Poppitz, Anomaly matching, (axial) Schwinger models and high-T super Yang-Mills domain walls, JHEP 09 (2018) 076 [arXiv: 1807.00093] [INSPIRE].

[52] M.M. Anber and E. Poppitz, Domain walls in high-T SU(N) super Yang-Mills theory and QCD(adj), JHEP 05 (2019) 151 [arXiv:1811.10642] [INSPIRE].

[53] G.W. Gibbons and C.N. Pope, $C P^{2}$ as a gravitational instanton, Commun. Math. Phys. 61 (1978) 239 [INSPIRE].

[54] T. Eguchi, P.B. Gilkey and A.J. Hanson, Gravitation, gauge theories and differential geometry, Phys. Rept. 66 (1980) 213 [INSPIRE].

[55] A. Kirchberg, J.D. Lange and A. Wipf, Extended supersymmetries and the Dirac operator, Annals Phys. 315 (2005) 467 [hep-th/0401134] [INSPIRE]. 